\title{
Angiopoietin-like 4 binds neuropilins and cooperates with VEGF to induce diabetic macular edema
}

\author{
Akrit Sodhi, ${ }^{1}$ Tao Ma, ${ }^{2}$ Deepak Menon, ${ }^{2}$ Monika Deshpande, ${ }^{1}$ Kathleen Jee, ${ }^{1}$ Aumreetam Dinabandhu, ${ }^{2}$ Jordan Vancel, ${ }^{1}$ \\ Daoyuan Lu, ${ }^{2}$ and Silvia Montaner ${ }^{2,3}$ \\ 'Wilmer Eye Institute, Johns Hopkins School of Medicine, Johns Hopkins University, Baltimore, Maryland, USA. Department of Oncology and Diagnostic Sciences, School of Dentistry, and \\ ${ }^{3}$ Greenebaum Cancer Center, University of Maryland, Baltimore (UMB), Maryland, USA.
}

\begin{abstract}
The majority of patients with diabetic macular edema (DME), the most common cause of vision loss in working-age Americans, do not respond adequately to current therapies targeting VEGFA. Here, we show that expression of angiopoietinlike 4 (ANGPTL4), a HIF-1-regulated gene product, is increased in the eyes of diabetic mice and patients with DME. We observed that ANGPTL4 and VEGF act synergistically to destabilize the retinal vascular barrier. Interestingly, while ANGPTL4 modestly enhanced tyrosine phosphorylation of VEGF receptor 2, promotion of vascular permeability by ANGPTL4 was independent of this receptor. Instead, we found that ANGPTL4 binds directly to neuropilin 1 (NRP1) and NRP2 on endothelial cells (ECs), leading to rapid activation of the RhoA/ROCK signaling pathway and breakdown of EC-EC junctions. Treatment with a soluble fragment of NRP1 (sNRP1) prevented ANGPTL4 from binding to NRP1 and blocked ANGPTL4-induced activation of RhoA as well as EC permeability in vitro and retinal vascular leakage in diabetic animals in vivo. In addition, sNRP1 reduced the stimulation of EC permeability by aqueous fluid from patients with DME. Collectively, these data identify the ANGPTL4/NRP/RhoA pathway as a therapeutic target for the treatment of DME.
\end{abstract}

\section{Introduction}

Human angiopoietin-like (ANGPTL) proteins are a family of 8 related gene products that are structurally similar to the angiopoietins (ANGPTs) and that play a role in a wide array of biological functions, including the regulation of lipid and glucose metabolism, hematopoietic stem cell expansion, chronic inflammation, angiogenesis, and wound healing $(1,2)$. ANGPTL proteins are characterized structurally by the presence of 2 major domains: an amino N-terminal coiled-coil domain and a C-terminal fibrinogen-like domain, the latter present in ANGPTL1-7, but not in ANGPTL8 $(1,3)$. Although ANGPTL proteins have been shown to participate in important endocrine, vascular, proliferative, and inflammatory processes, the molecular signaling events that explain their mechanisms of action are still largely unknown.

One member of the ANGPTL family, ANGPTL4, has been implicated in many pathological disorders, including cardiac and lung diseases, tumor development and dissemination, joint diseases, diabetes, atherosclerosis, and nephrotic syndrome $(4,5)$. ANGPTL4 is a multifunctional circulating protein that undergoes posttranslational modifications (glycosylation) and subsequent proteolytic processing by membrane proprotein convertases upon secretion $(6,7)$. The ANGPTL4 N-terminal coiled-coil domain (nANGPTL4) inhibits lipoprotein lipase (LPL), the enzyme responsible for the hydrolysis of circulating triglycerides (TGs) into free

Conflict of interest: The authors have declared that no conflict of interest exists. Copyright: () 2019, American Society for Clinical Investigation.

Submitted: March 6, 2018; Accepted: August 1, 2019; Published: September 23, 2019. Reference information: J Clin Invest. 2019;129(11):4593-4608.

https://doi.org/10.1172/JCl120879. fatty acids, under conditions of fasting and exercise (7-9). Because of this role in TG metabolism, LPL has been suggested as a therapeutic target for reducing plasma lipids. Interestingly, human genetics studies using carriers of a missense E40K variant of ANGPTL4 or other inactivating ANGPTL4 mutations showed lower levels of TGs and higher levels of plasma high-density lipoprotein (HDL) cholesterol and lower risk of coronary artery disease (CAD) compared with control individuals expressing WT ANGPTL4 (10). As modulation of LPL activity affects free fatty acid delivery, nutrient partitioning, and insulin sensitivity (11), inactivating ANGPTL4 mutations are also associated with lower fasting glucose levels, greater insulin sensitivity, and reduced risk of type 2 diabetes (12). These genetic studies demonstrate the metabolic benefits and therapeutic potential of reducing plasma ANGPTL4 levels for the treatment of metabolic disorders.

Alternatively, the ANGPTL4 C-terminal domain (cANGPTL4) appears to have an important role in anoikis resistance, altered redox regulation, angiogenesis, and vascular hyperpermeability $(4,5)$. This C-terminal sequence of ANGPTL4 is shorter than the corresponding homologous regions within the distantly related proteins ANGPT1 and ANGPT2; as a consequence, cANGPTL4 cannot bind to the vascular endothelial cell (EC) TIE1 or TIE2 receptors (13), which are required for ANGPT1 and ANGPT2 to regulate the blood-retinal barrier in diabetic retinopathy (14-20).

Despite the absence of a binding partner for ANGPTL4 on vascular ECs, emerging evidence supports the role of ANGPTL4 in vascular biology. Specifically, it has been reported that ANGPTL4 promotes EC migration and angiogenesis (21) and induces the destabilization of the EC-EC tight junctions (TJs) and adherens junctions (AJs) that preserve the integrity of the vascular barrier 


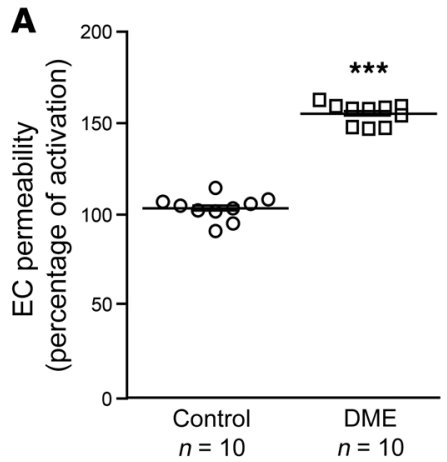

B
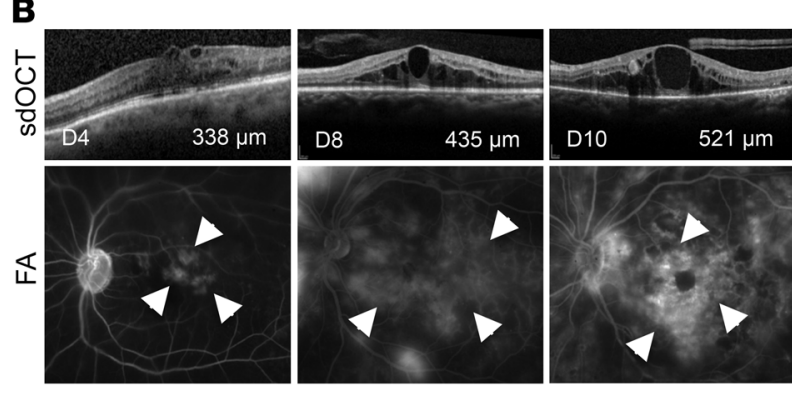

C

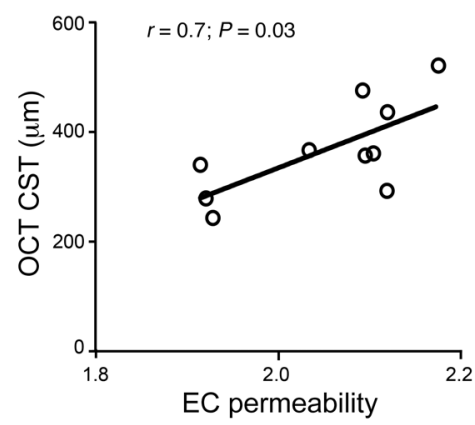

D

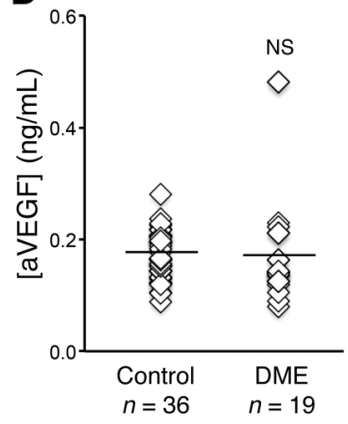

E

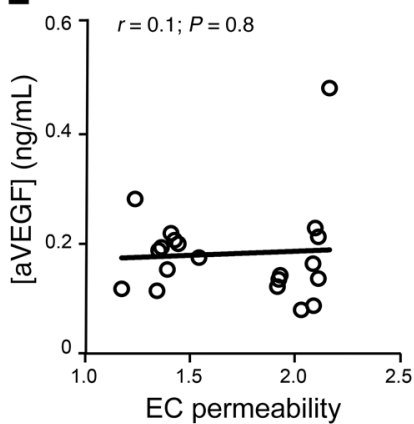

$\mathbf{F}$

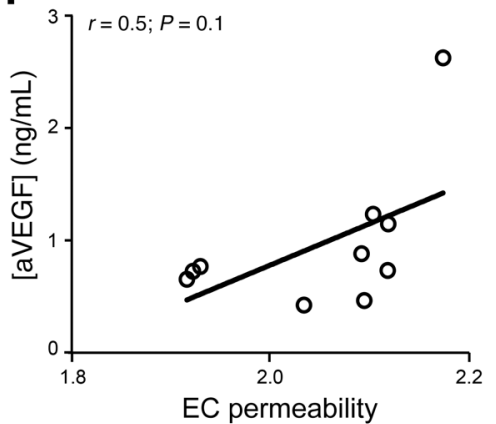

G

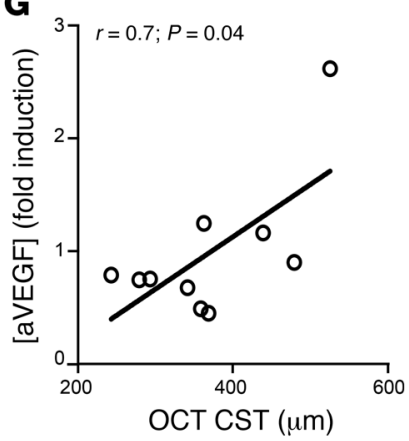

Figure 1. Vasoactive factors in the aqueous fluid from DME patients besides VEGF promote EC permeability in vitro and correlate with ME in patients. (A) Induction of in vitro EC permeability by aqueous samples from nondiabetic (control) patients and diabetic patients with active DME (see Supplemental Table 1). DME patients show a 52\% mean increase in the induction of EC permeability compared with control patients. (B) Correlation of vascular permeability with the CST on sdOCT for 3 representative DME patients (D4, D8, D10). FA, fluorescein angiogram. Arrowheads indicate leakage of fluorescein dye from retinal vasculature. (C) Correlation of the promotion of EC permeability by aqueous fluid from DME patients with their CST on sdOCT, $r=0.7 ; P=0.03$. (D) Levels of VEGF in aqueous samples from nondiabetic (control) patients and diabetic patients with DME who have not previously received anti-VEGF therapy or have not received anti-VEGF therapy for 12 weeks or longer in the sample eye (see Supplemental Table 2) (E) Correlation of EC permeability with levels of VEGF in aqueous samples from diabetic patient with DME. $r=0.1 ; P=0.8$ (E). (F and $\mathbf{G})$ Correlation of EC permeability $(r=0.5 ; P=0.1)(\mathbf{F})$ and $C S T$ on sdOCT ( $r=0.7 ; P=0.04$ ) (G) with levels of VEGF in aqueous samples from diabetic patients with DME (see Supplemental Table 2). Two-tailed unpaired Student's $t$ test (A), Mann-Whitney $U$ test (D), Pearson's correlation (C, E, F, G). ${ }^{* * *} P<0.001$.

(21-24). In cancer, release of cANGPTL4 by tumor cells induces tumor growth, venous invasion, and metastasis (4). In addition, a role of cANGPTL4 as a diagnostic and/or prognostic marker of cancer progression has been proposed for different solid tumors, including colon cancer (25), gastric cancer (26), breast cancer (27), prostate cancer (28), hepatocellular carcinoma $(29,30)$, head and neck squamous cell carcinoma (31-34), Kaposi's sarcoma $(21,35)$, and renal cell carcinoma $(36,37)$.

More recently, we have reported a role for ANGPTL4 in ischemic retinal disease. We have observed that ANGPTL4 is a critical retinal hyperpermeability factor and that its expression is increased in response to the accumulation of the transcription factor HIF-1 in hypoxic retinal Müller glial cells in animal models of ischemic retinopathies as well as in patients with ischemic retinal disease $(24,38-40)$. In these settings, the aberrant expression of ANGPTL4 and other vasoactive mediators can lead to vascular hyperpermeability and accumulation of interstitial fluid in the macula, culminating in the development of macular edema (ME), the major cause of severe vision loss in the American working-age population (41-43). Over the last decade, therapies targeting another HIF-regulated vasoactive mediator, VEGFA, have had a profound impact on the treatment of patients with ME (44). As a consequence, intraocular injections with "anti-VEGF" therapies are currently the gold standard for the treatment of diabetic macular edema (DME). Nonetheless, despite strict adherence to monthly anti-VEGF injections, most patients with DME do not demonstrate a clinically significant improvement in vision (defined as 3 or more lines on the ETDRS vision chart), suggesting that other vasoactive factors may also contribute to its development. In this regard, we have recently proposed that expression of ANGPTL4 in the eyes of patients with diabetic eye disease may help explain why some patients do not respond adequately to monthly treatment with anti-VEGF therapies (45).

Despite accumulating and compelling data supporting a role for ANGPTL4 in the pathogenesis of ischemic retinopathies, little is known about the molecular events that mediate its biological effects. And whether the signaling pathways activated by ANGPTL4 overlap with - or are independent of - VEGFA remains unclear. To identify new approaches for the treatment of $\mathrm{ME}$, we explore here the molecular events whereby ANGPTL4 promotes vascular permeability to exert its pathological effects in diabetic eye disease.

\section{Results}

Vasoactive factors in the aqueous fluid from DME patients, besides $V E G F$, promote EC permeability in vitro and correlate with $M E$ in patients. To quantitate the promotion of vascular hyperperme- 
A

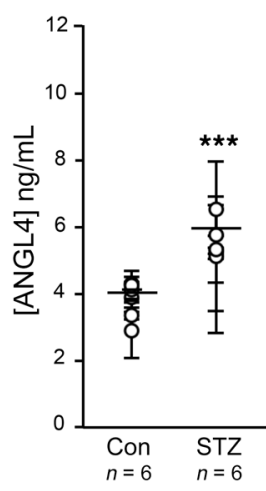

B

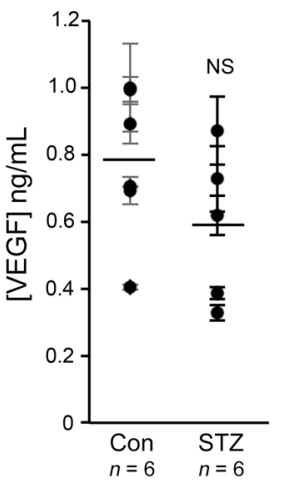

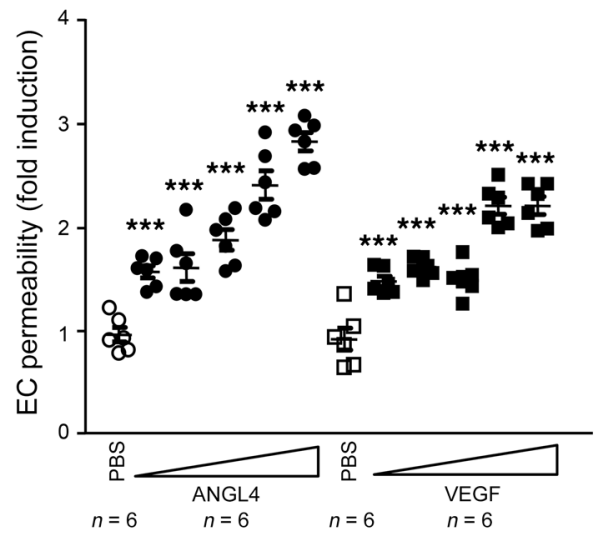

hREC

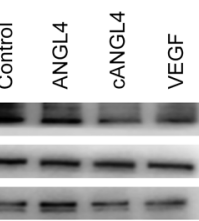

$\mathbf{F}$

Claudin 5

Occludin

VE cadherin

$\beta$-Catenin

Actin PBS

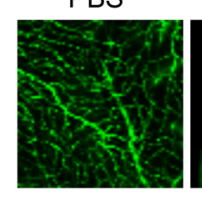

ANGL4
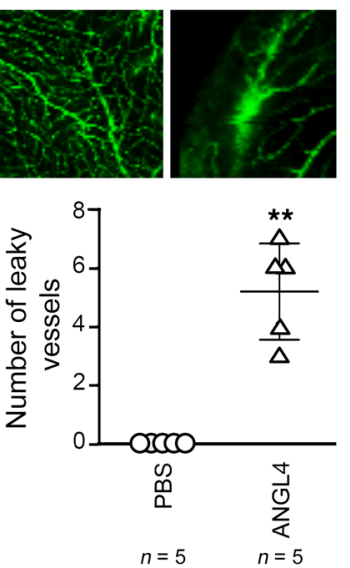

C
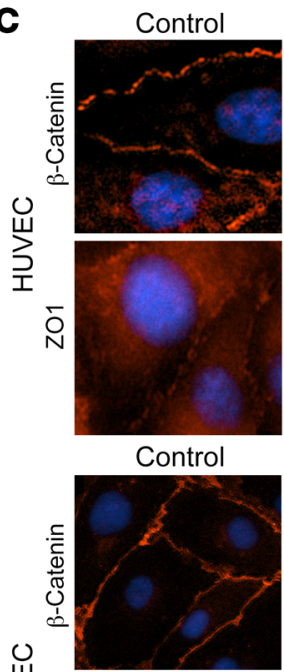

崩

ANGL4

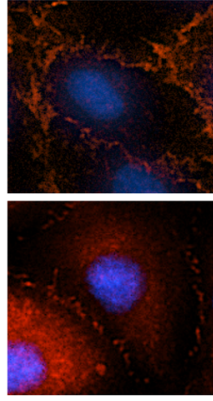

ANGL4

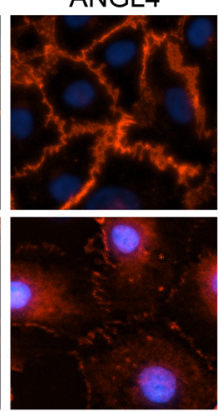

G

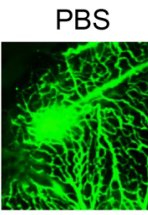

VEGF

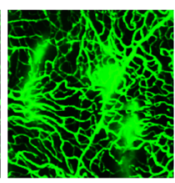

ANGL4

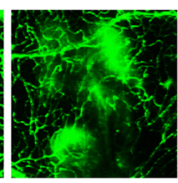

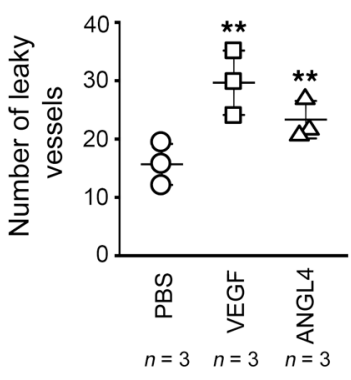

Figure 2. ANGPTL4 is increased in diabetic STZ mice and promotes EC permeability in vitro and retinal vascular leakage in vivo. (A) ANGPTL4 and VECF protein levels in control and diabetic STZ animals with sustained hyperglycemia for 3 months. (B) Dose-response curve of the induction of EC permeability by rhANGPTL4 $(0.5,1,2,5,10 \mu \mathrm{g} / \mathrm{mL})$ or rhVEGFA $(1,5,10,50,100 \mathrm{ng} / \mathrm{mL})$ in HUVECs. (C) Destabilization of the integrity of vascular Als $(\beta$-catenin staining) and TJs (ZO1 staining) of HUVEC or hREC monolayers treated for 6 hours with PBS (control) or rhANGPTL4 (5 $\mu \mathrm{g} / \mathrm{mL})$. Original magnification, $\times 20$. (D and E) Changes in AJ and TJ protein levels upon treatment of HUVEC (D) or hREC (E) with rhANGPTL4 ( $5 \mu \mathrm{g} / \mathrm{mL})$, rhcANGPTL4 (5 $\mu \mathrm{g} / \mathrm{mL})$, or rhVECF ( $50 \mathrm{ng} /$ $\mathrm{mL}$ ) for 24 hours. (F and $\mathbf{G}$ ) Induction of retinal vascular permeability by intraocular injection of C57BL/6) mice (F) or diabetic, STZ mice (C) with $1 \mu$ l of PBS, rmVEGFA $(100 \mathrm{ng} / \mu \mathrm{l})$ or rmANGPTL4 $(200 \mathrm{ng} / \mu \mathrm{l})$. Original magnification, $\times 20$. Two-tailed unpaired Student's $t$ test $(\mathbf{A}, \mathbf{F}, \mathbf{G}), 1-$ way ANOVA (B). ${ }^{* *} P<0.01$; ${ }^{* * *} P<0.001$. Experiments were repeated at least 3 times.

ability in diabetic eyes, we obtained aqueous fluid from diabetic patients with DME (Supplemental Table 1; supplemental material available online with this article; https://doi.org/10.1172/ JCI120879DS1) and performed EC permeability assays in vitro. We observed a marked increase in the promotion of EC permeability upon exposure of HUVECs to aqueous fluid from patients with DME compared with nondiabetic (control) patients (Figure 1A). Vascular permeability, assessed clinically by vascular leakage on fluorescein angiography, correlated with DME, assessed clinically by the central subfield thickness (CST) on spectral domain optical coherence tomography (sdOCT; Figure 1B) (46). Impor- tantly, the promotion of EC permeability by aqueous fluid from DME patients also correlated with the CST on sdOCT (Figure 1C; $r=0.7, P=0.03$ ), suggesting that measurements of the effects of vasoactive factors in aqueous fluid reflect their pathologic effects in the eyes of diabetic patients.

Surprisingly, the levels of VEGFA, a well-described pathogenic hyperpermeability factor in DME development, were not significantly elevated in aqueous fluid from DME patients compared with that from nondiabetic control patients (Supplemental Table 2 and Figure 1D). These DME patients included untreated patients without previous anti-VEGF therapy and previously treat- 

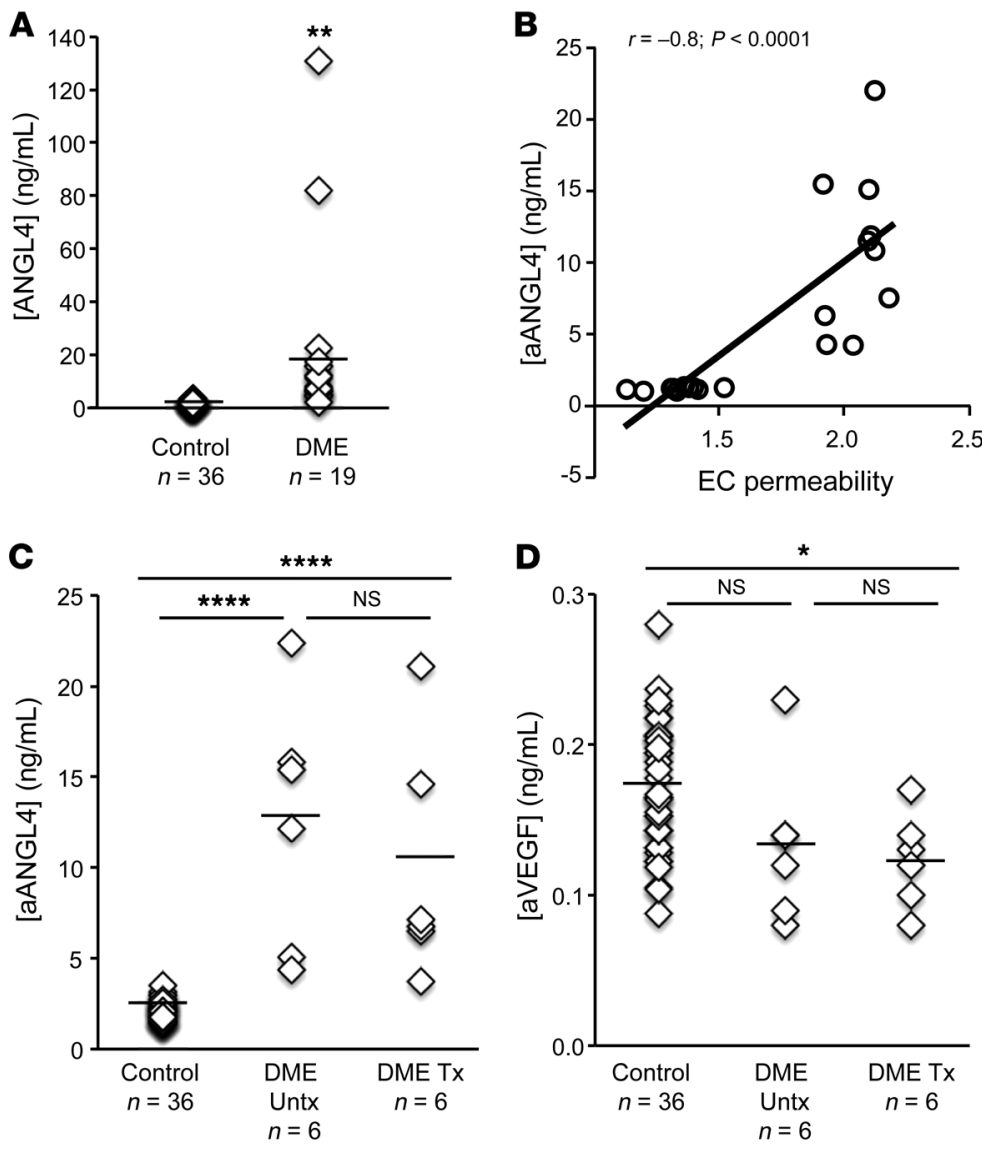

Figure 3. Upregulation of ANGPTL4 in patients with DME. (A and $\mathbf{B}$ ) Levels of ANGPTL4 in aqueous samples from nondiabetic (control) patients and diabetic patients with DME who have not previously received anti-VEGF therapy or have not received anti-VEGF therapy for 12 weeks or longer in the sample eye (see Supplemental Table 2) (A) and its correlation with EC permeability. $r=-0.8 ; P<0.0001$ (B). (C and D) ANGPTL4 (C) and VEGF (D) protein levels in DME patients without previous treatment with anti-VEGF therapy (DME Untx) or DME patients who have received a single treatment with anti-VEGF therapy in the sample eye within 6 weeks of sample collection (DME Tx) compared with nondiabetic (control) patients. Mann-Whitney $U$ test (A), Pearson correlation (B), or 1-way ANOVA (C and D). ${ }^{*} P<0.05$; ${ }^{* *} P<0.01 ;{ }^{* * *} P<0.0001$.

increased vascular permeability, similar to that observed in patients with diabetic eye disease (47). Upon 3 months of sustained hyperglycemia, we observed a marked increase in ANGPTL4 in the diabetic mice compared with that in control mice (Figure 2A). However, we did not see an increase in the levels of VEGFA.

To assess the contribution of ANGPTL4 to the promotion of vascular leakage and ME, we treated HUVEC monolayers with recombinant human ANGPTL4 (rhANGPTL4) and observed an increase in EC permeability, similar to that observed using rhVEGFA (Figure $2 \mathrm{~B}$ ). We next assessed the destabilization of the integrity of vascular AJs and TJs by ANGPTL4 by examining the cellular localization of the corresponding component of these junctions, $\beta$-catenin and zonula occludens 1 (ZO1),

ed patients who had not received anti-VEGF therapy for 12 weeks or longer. Accordingly, we did not observe a correlation between the levels of VEGF in the aqueous fluid of patients and the promotion of vascular permeability (Figure $1 \mathrm{E} ; r=0.1 ; P=0.8$ ). However, when we looked only at aqueous fluid from diabetic patients, we did observe a modest trend toward a correlation between levels of VEGF and the promotion of vascular permeability (Figure $1 \mathrm{~F} ; r=$ $0.5, P=0.1$. Indeed, the aqueous fluid levels of VEGF in diabetic patients did correlate with CST on sdOCT (Figure 1G; $r=0.7, P=$ 0.04). Collectively, these results suggest that additional hyperpermeability factors may directly contribute to the promotion of EC permeability by aqueous fluid from DME patients and further help unmask the vasoactive potential of VEGF in these patients.

ANGPTL4 is increased in diabetic streptozotocin mice and promotes destabilization of EC-EC junctions and permeability in vitro and vascular leakage in vivo. Many patients with DME do not respond adequately to therapies targeting VEGFA, further supporting a role for other vasoactive factors in the promotion of vascular permeability in diabetic eye disease. We have previously reported that expression of a second HIF-regulated vasoactive mediator, ANGPTL4, is increased in the oxygen-induced retinopathy (OIR) mouse model for ischemic retinal disease as well as in patients with proliferative diabetic retinopathy $(24,38)$. To assess whether ANGPTL4 contributes to the development of DME, we first examined its expression in the streptozotocin (STZ) mouse model for hyperglycemia. In this model, sustained hyperglycemia results in secondary injury of the retinal microvasculature and using immunofluorescence microscopy (Figure 2C). HUVEC and primary human retinal EC (hREC) monolayers treated with rhANGPTL4 showed a more diffuse and discontinuous localization of $\beta$-catenin and ZO1 at cell-cell contacts, consistent with breakdown in the integrity of these critical EC-EC junctions. We then determined whether ANGPTL4 could induce a change in the expression levels of $\mathrm{AJ}$ and TJ proteins. For these assays, we used full-length ANGPTL4 (rhANGPTL4) as well as the ANGPTL4 C-terminal domain (rhcANGPTL4), as this latter fragment was previously reported to play a role in vessel leakage (21-24), and looked at changes in ZO1, ZO2, ZO3, claudin 5, occludin, VE-cadherin, and $\beta$-catenin expression (Figure 2, D and E). We observed a decrease in the levels of ZO1, ZO3, occludin, and VE-cadherin upon treatment of HUVECs for 24 hours with ANGPTL4, cANGPTL4, or VEGFA (Figure 2D and Supplemental Figure 1). We also observed a decrease in ZO1 and occludin levels when we treated hRECs with these factors (Figure 2E and Supplemental Figure 2).

The promotion of EC permeability in vitro by ANGPTL4 was corroborated in vivo, using intraocular injection with recombinant murine ANGPTL4 (rmANGPTL4) in C57BL/6J mice, which led to retinal vascular leakage (Figure $2 \mathrm{~F}$ ). This effect was markedly increased in mice that were hyperglycemic for 3 months to mimic the underlying damage to the retinal microvasculature observed with sustained hyperglycemia in diabetic patients (Figure 2G). Collectively, these experiments demonstrate that ANGPTL4 regulates the integrity of EC-EC junctions, promotes increased vascular permeability, and in turn, could contribute to the development of DME. 

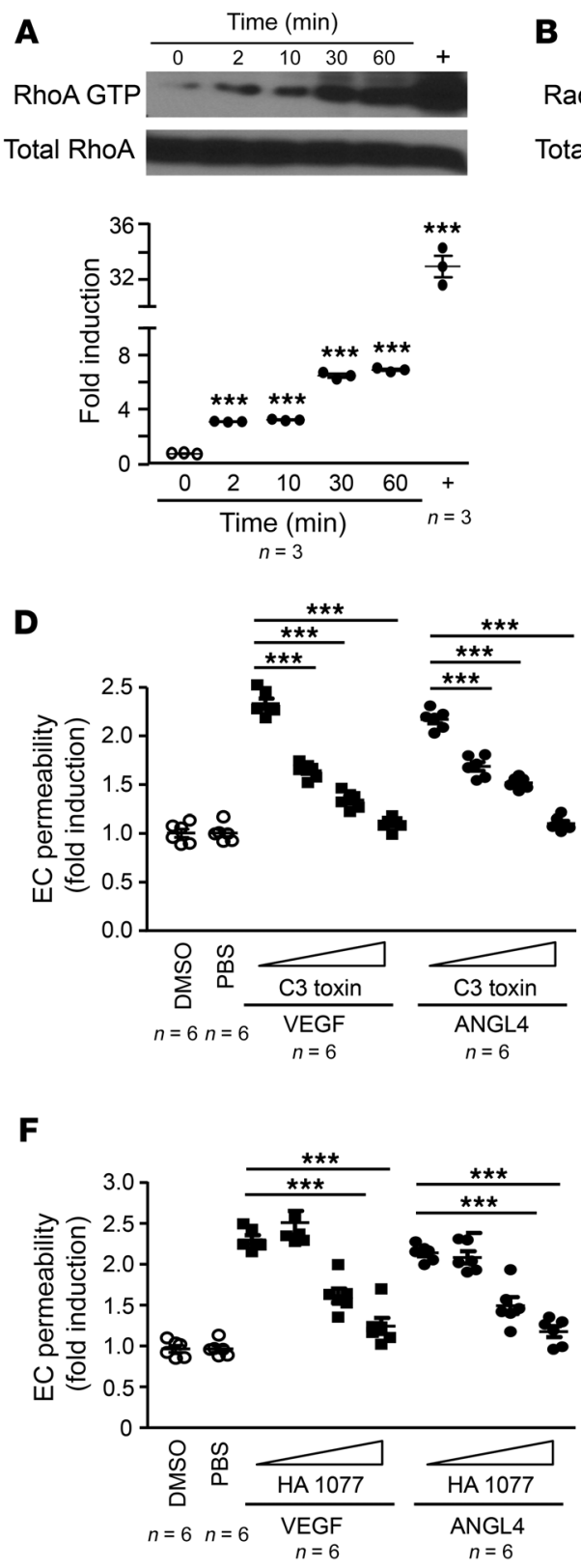

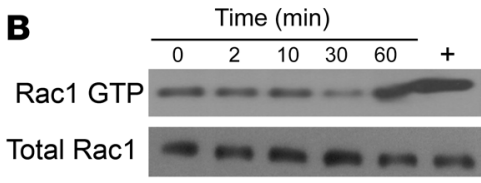

C
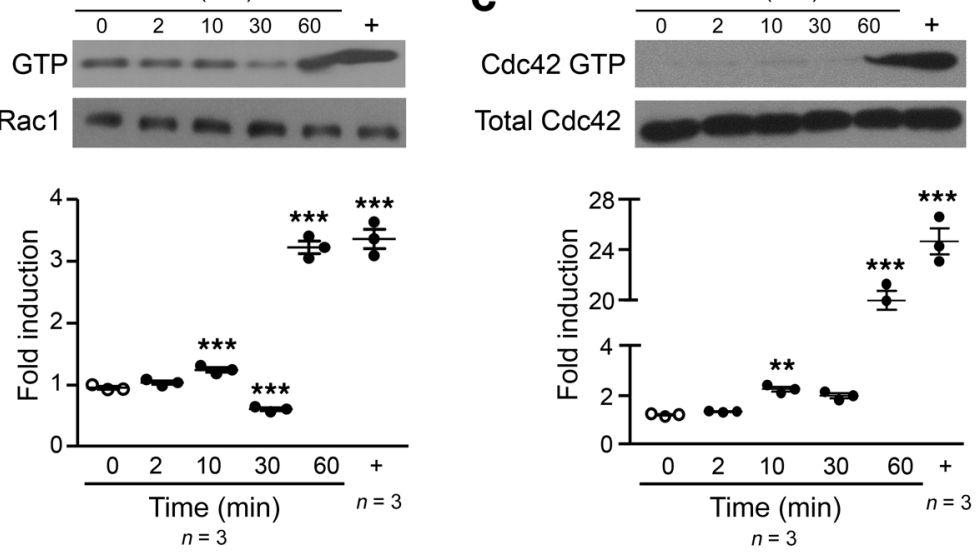
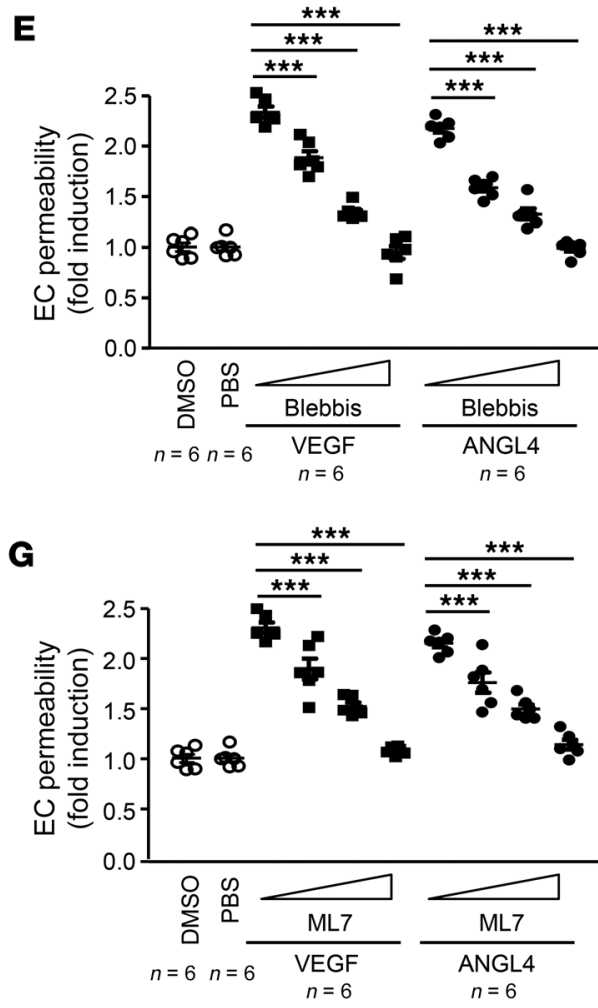

Figure 4. ANGPTL4 induces a rapid activation of RhoA and actomyosin contraction and a delayed activation of Rac1 and Cdc42. (A-C) Activation of RhoA (A), Rac1 (B), and Cdc42 (C) over time in HUVECs exposed to $5 \mu \mathrm{g} / \mathrm{mL}$ rhANGPTL4. Positive controls: $0.5 \mathrm{U} / \mathrm{mL}$ thrombin (Rho assay); $1 \mu \mathrm{M}$ sphingosine-1-phosphate (Rac1 and Cdc42 assays). Plus signs indicate positive controls. (D-G) Inhibition of $5 \mu \mathrm{g} / \mathrm{mL}$ rhANGPTL4-induced in vitro EC permeability by increasing doses of $C$. botulinum $\mathrm{C3}$ toxin $(0,0.5,2,5 \mu \mathrm{g} / \mathrm{mL})(\mathbf{D}), \mathrm{HA} 1077(0,0.1,1,10 \mu \mathrm{M})(\mathbf{E})$, blebbistatin $(0,0.5,5,10 \mu \mathrm{M})(\mathbf{F})$, and ML7 (0, 10, 50 , $100 \mu \mathrm{M})(\mathbf{G})$. One-way ANOVA (A-G). ${ }^{* *} P<0.01 ;{ }^{* * *} P<0.001$. Experiments were repeated at least 3 times.

ANGPTL4 is increased in diabetic eyes. We next measured ANGPTL4 protein levels in aqueous fluid samples obtained from patients with active DME (Supplemental Table 2). We observed a marked (9-fold) increase in the levels of ANGPTL4 in patients with DME $(19.72 \pm 32.05 \mathrm{ng} / \mathrm{mL} ; n=36)$ compared with that in nondiabetic control patients $(2.18 \pm 0.53 \mathrm{ng} / \mathrm{mL} ; n=19)$ (Figure $3 \mathrm{~A})$. Accordingly, there was a strong correlation between the ability of aqueous fluid to promote EC permeability and the levels of ANGPTL 4 in these samples (Figure $3 \mathrm{~B} ; r=-0.8, P<0.0001$ ).

Importantly, when we checked the levels of ANGPTL4 in patients with DME who received anti-VEGF therapy in the sample eye within 6 weeks of sample collection (DME Tx) compared with DME patients who had never previously received anti-VEGF therapy (DME Untx), we observed that ANGPTL4 remained elevated in treated patients (Figure $3 \mathrm{C}$ ). Aqueous VEGF levels were not elevated in the same, recently treated (i.e., within 6 weeks) DME patients; moreover, there was a significant decrease in VEGF levels following treatment with anti-VEGF therapy (Figure 3D). This suggests that expression of ANGPTL4 is independent of VEGFA expression and that current therapies targeting VEGFA do not affect the levels of ANGPTL4. Collectively, these findings support a role for ANGPTL4 in the pro- 
A

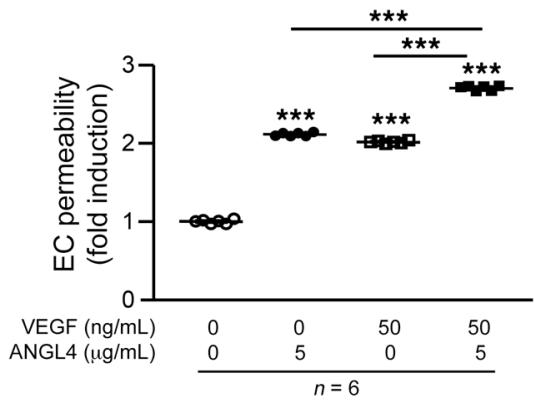

B

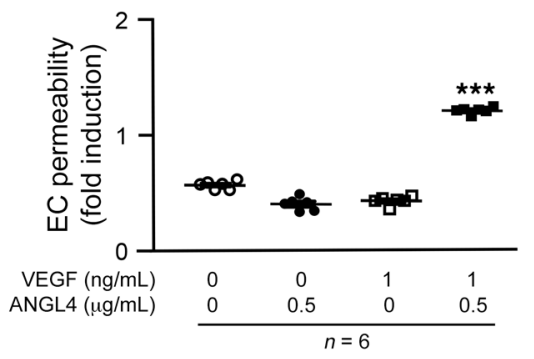

D

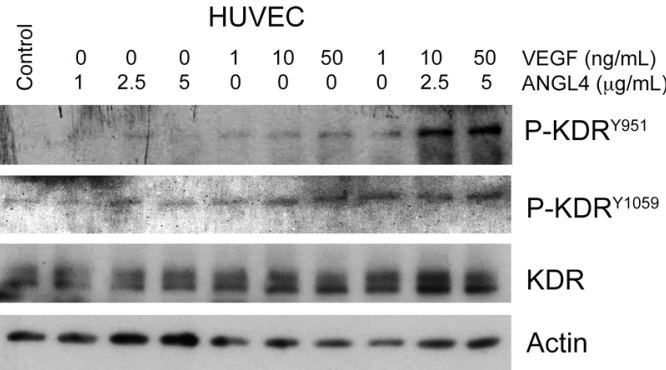

C
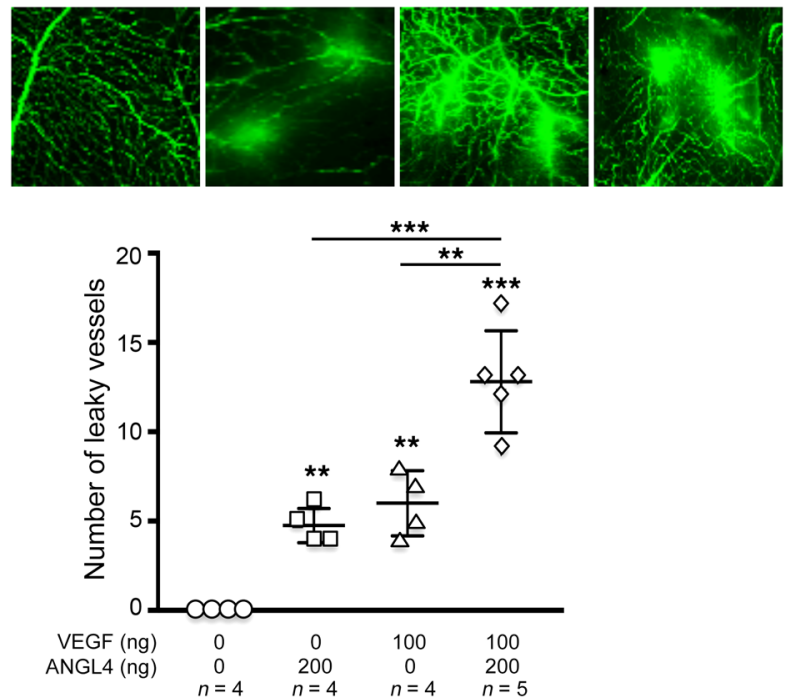

E

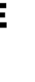

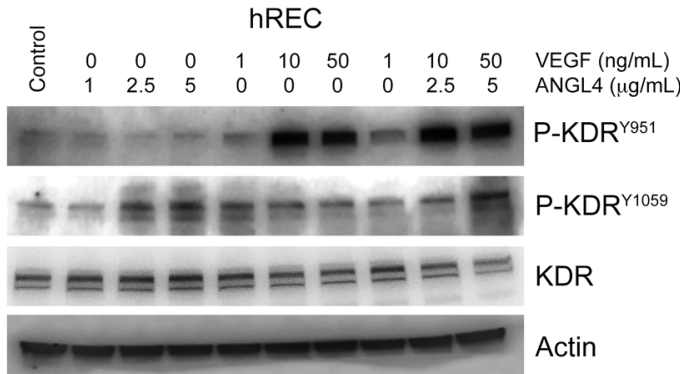

H

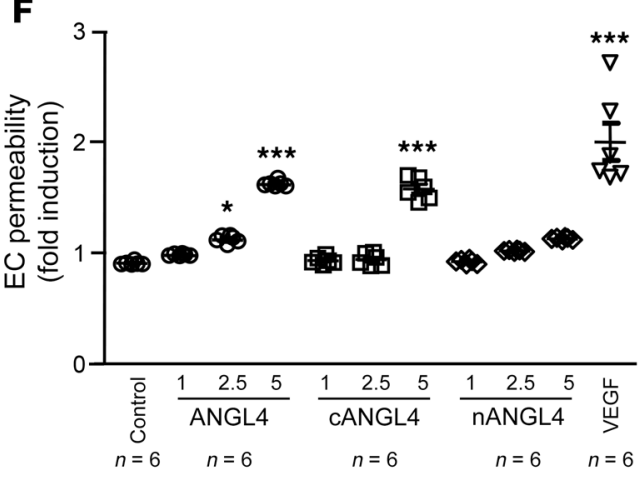

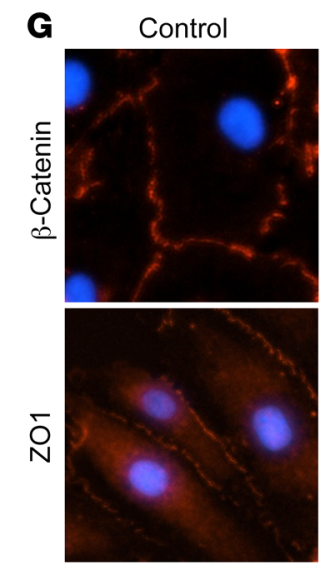

CANGL4
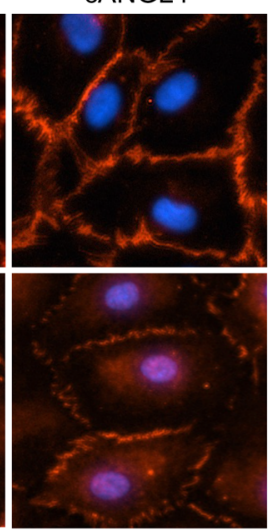

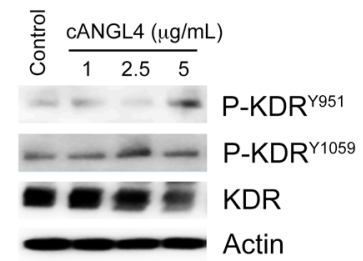

Figure 5. ANGPTL4 and VEGF cooperate in the induction of the increase in retinal vascular permeability. (A and B) EC permeability assay upon treatment of HUVECs with optimal (A) or suboptimal (B) concentrations of rhANGPTL4, rhVEGFA, or both. (C) Vascular permeability assay following intraretinal injection with $1 \mu$ l of PBS or rmVEGFA, rmANGPTL4, or both. (D and E) KDR phosphorylation on Tyr951 and Tyr1059 upon treatment of HUVECs (D) or hREC (E) with different doses of rhANGPTL4 $(\mu \mathrm{g} / \mathrm{mL})$, rhVEGFA $(\mathrm{ng} / \mathrm{mL})$, or both. (F) EC permeability assay upon treatment with rhANGPTL4, rhcANGPTL4, rhnANGPTL4 ( $\mu \mathrm{g} / \mathrm{mL})$, or none (control) in HUVECs. VEFCA $(50 \mathrm{ng} / \mathrm{mL})$ served as a control. (G) Destabilization of the vascular AJs ( $\beta$-catenin staining) and TJs (ZO1 staining) of hREC monolayers treated for 6 hours with PBS (control) or rhcANGPTL4 $(5 \mu \mathrm{g} / \mathrm{mL})$. Original magnification, $\times 20$. (H) KDR phosphorylation in Tyr951 and Tyr1059 upon treatment of HUVECs with rhcANGPTL4 $(\mu \mathrm{g} / \mathrm{mL})$. One-way ANOVA (A, B, C, F). ${ }^{* *} P<0.01 ;{ }^{* *} P<0.001$. Experiments were repeated at least 3 times.

motion of DME, including in patients who do not respond adequately to anti-VEGF therapies.

ANGPTL4-induced vascular permeability is mediated by sequential activation of RhoA and Rac1/Cdc42 small GTPases. We next set out to investigate the mechanism whereby ANGPTL4 promotes retinal vessel hyperpermeability. In this regard, it is known that the 3 small g proteins, RhoA, Rac1, and Cdc42, are master regulators of the actin cytoskeleton and the mechanisms that control cell motility and intercellular adhesion (48). Interestingly, when we treated HUVECs with rhANGPTL4, we 
A

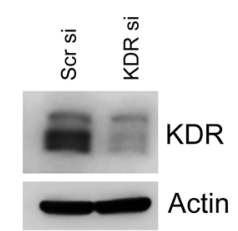

D

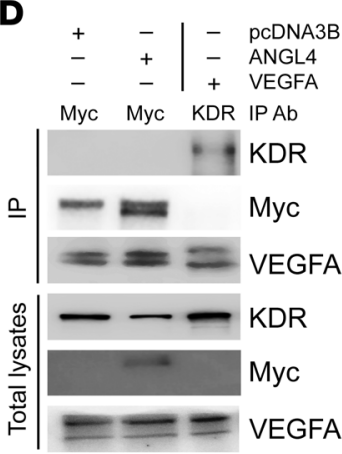

B

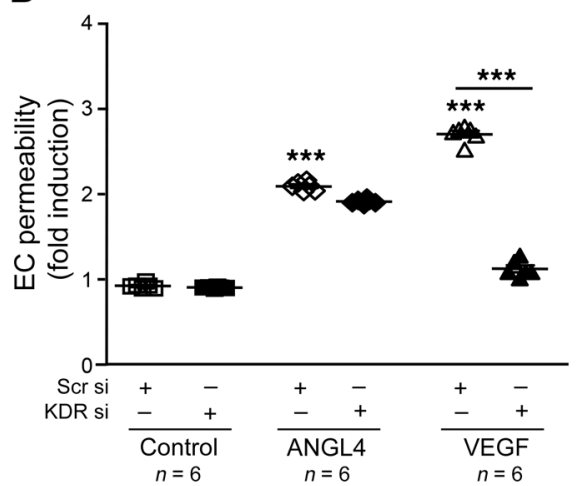

E

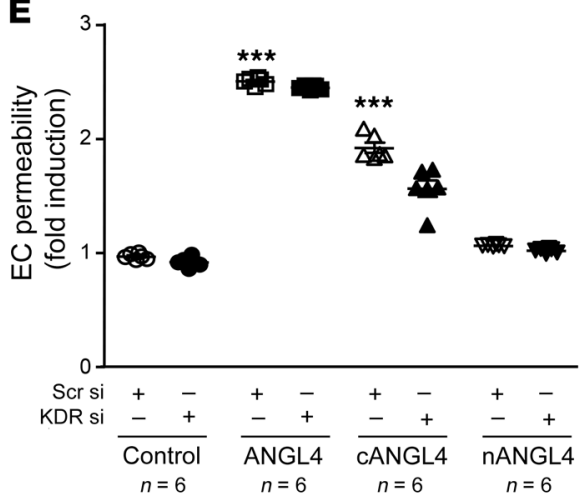

C

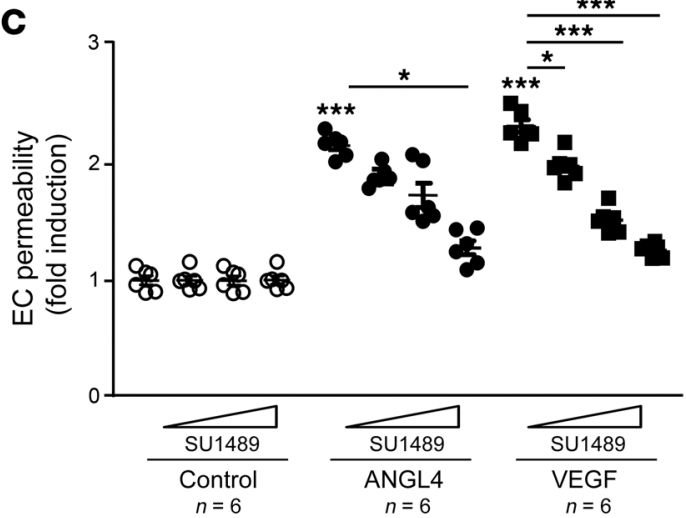

$\mathbf{F}$

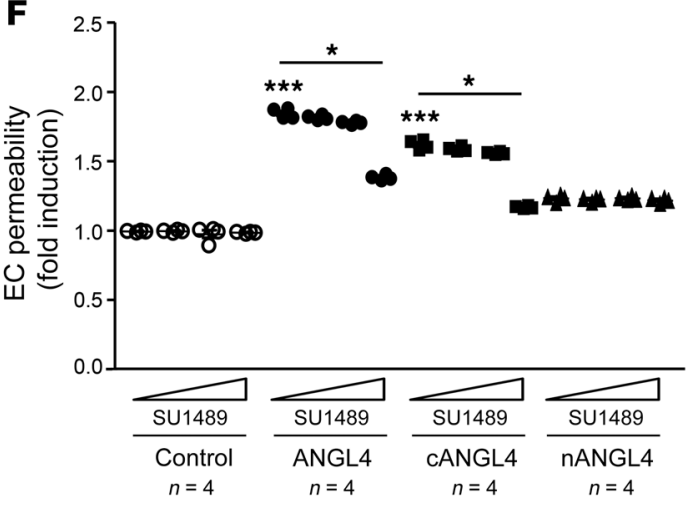

Figure 6. KDR is not required for ANGPTL4 promotion of EC permeability. (A) HUVECs were transfected with $50 \mathrm{nM}$ scrambled (Scr) siRNA (si) or $50 \mathrm{nM}$ KDR siRNA. Inhibition of KDR expression using Western blot is shown. (B) EC permeability assay upon transfection of scrambled siRNA or KDR siRNA and treatment with $(5 \mu \mathrm{g} / \mathrm{mL})$ rhANGPTL4 or $(50 \mathrm{ng} / \mathrm{mL})$ rhVEGFA or no treatment (control) in HUVECs. (C) EC permeability assay upon treatment with 5 $\mu \mathrm{g} / \mathrm{mL}$ rhANGPTL4 or $50 \mathrm{ng} / \mathrm{mL}$ rhVEGFA or no treatment (control) of HUVECs pretreated (30 minutes) with SU1489. (D) Lack of immunoprecipitation of endogenous KDR with ANGPTL4 upon transfection of pcDNA3.1-ANGPTL4-mycHis in HUVECs. Transfection of pcDNA3.1-VEGFA is used as control. (E) EC permeability assay upon transfection of scrambled siRNA or KDR siRNA and treatment with $5 \mu \mathrm{g} / \mathrm{mL}$ rhANGPTL4, rhcANGPTL4,or rhnANGPTL4 or none (control) in HUVECs. (F) EC permeability upon treatment with $(5 \mu \mathrm{g} / \mathrm{mL})$ rhANGPTL4, rhcANGPTL4, or rhnANGPTL4 or none (control) in HUVECs pretreated (30 minutes) with SU1489. For SU1489 dose response, cells were pretreated with 0.01. 0.1, or $1 \mu \mathrm{M}$ of drug (black bars) compared with vehicle (white bars). One-way ANOVA (B, C, E, F). ${ }^{*} P<0.05 ;{ }^{* *} P<0.001$. Experiments were repeated at least 3 times.

observed an increase of the GTP-bound (active) forms of all 3 small g proteins (21) (Figure 4, A-C). However, while the activation of RhoA (Figure $4 \mathrm{~A}$ ) in treated ECs was rapid (within minutes), activation of Rac1 and Cdc42 GTPases was delayed (Figure 4, B and C), peaking 60 minutes after treatment. This late activation of Rac1/Cdc42 occurs after the induction of EC hyperpermeability by rhANGPTL4, which is observed within 30 minutes of treatment (Figure 2C).

When we treated HUVECs with rhANGPTL4 in the presence of pharmacologic inhibitors of RhoA (Clostridium botulinum C3 toxin; Figure 4D), Rho kinase (HA1077; Figure 4E), myosin II (Blebbistatin; Figure 4F), or myosin light chain (MLC) kinase (ML7 inhibitor; Figure 4G), all downstream effectors of the RhoA pathway (49), we found a dose-dependent inhibition of ANGPTL4induced EC permeability. These results support a role for the activation of RhoA and the RhoA-dependent contraction of actomyosin fibers in the loss of vascular integrity induced by this factor. Collectively, these results suggest that ANGPTL4 induces vascular hyperpermeability by rapidly inducing RhoA/Rho kinasedependent activation of actomyosin contractility, resulting in the destabilization of the EC-EC junctions.
ANGPTL4 and VEGF cooperate in the induction of the increase in retinal vascular permeability. The overlap in function of ANGPTL4 and VEGF prompted us to investigate whether these factors have a cooperative - or redundant - impact on destabilizing the integrity of the EC junctions and the promotion of vascular permeability. To this end, we simultaneously treated HUVECs with both rhANGPTL4 and rhVEGFA, and observed an additive effect on EC permeability (Figure 5A). Interestingly, when we titered down the concentrations of these 2 factors to concentrations at which no effect was found with either factor alone, we observed a synergistic promotion of EC permeability when the 2 factors were combined (Figure 5B). This cooperative effect of ANGPTL4 and VEGFA in the promotion of retinal hyperpermeability was observed in vivo following intraocular injections with suboptimal concentrations of both rmANGPTL4 and rmVEGFA in C57BL/6J mice (Figure 5C). Collectively, these results strongly support a cooperative - nonredundant - role for ANGPTL4 and VEGF in the promotion of vascular leakage in the retina.

We next explored whether the promotion of vascular permeability by ANGPTL4 and VEGF are interdependent. In this regard, it is known that activation of VEGFA signal transduction is medi- 
A

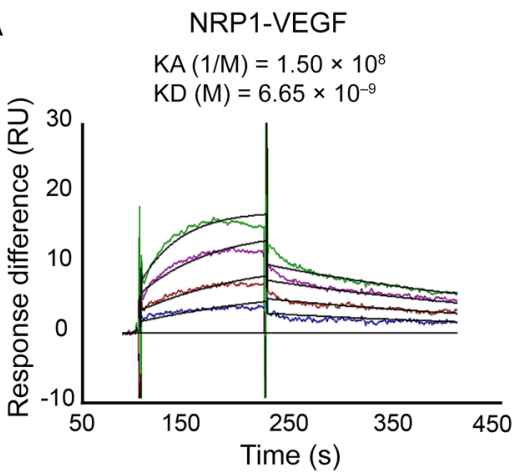

D

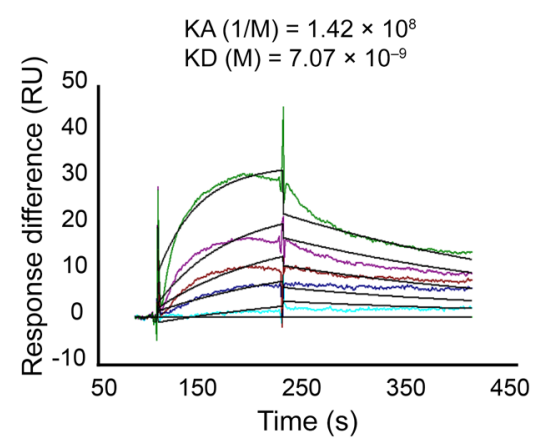

B

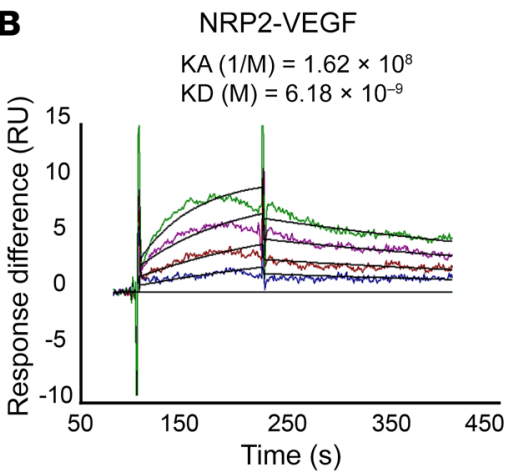

C NRP1-ANGL4

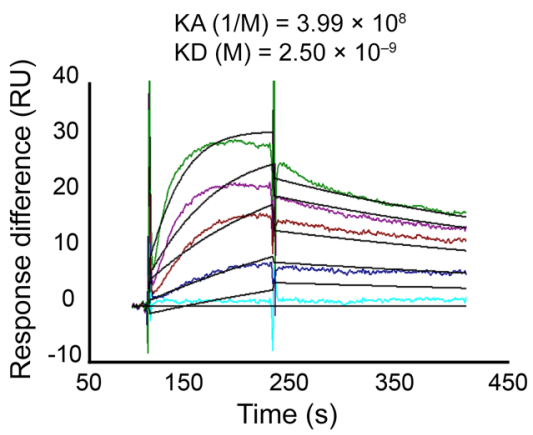

E

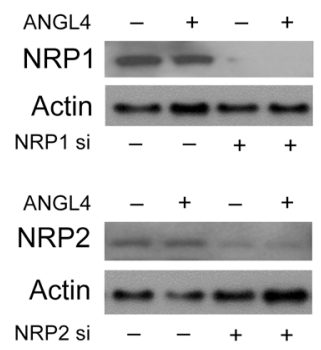

$\mathbf{F}$

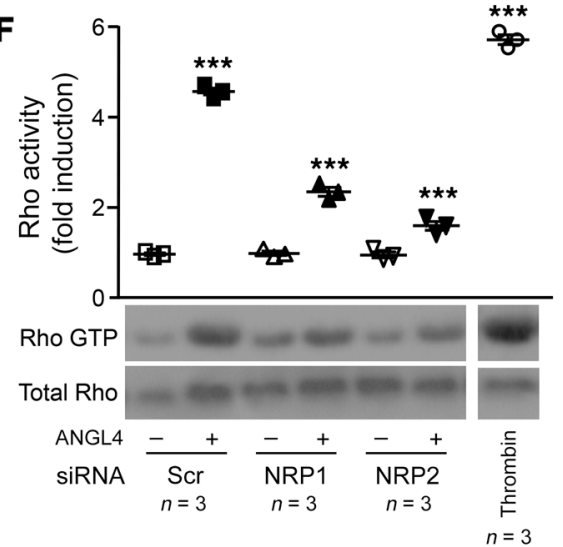

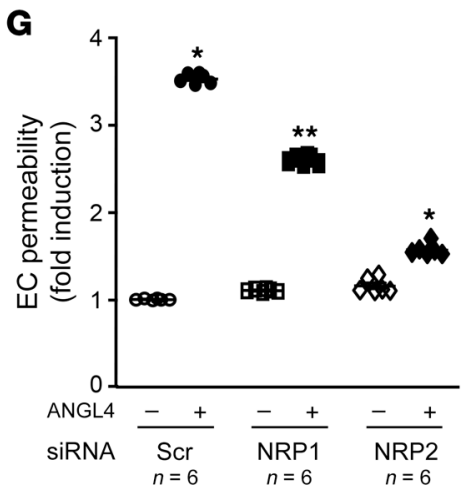

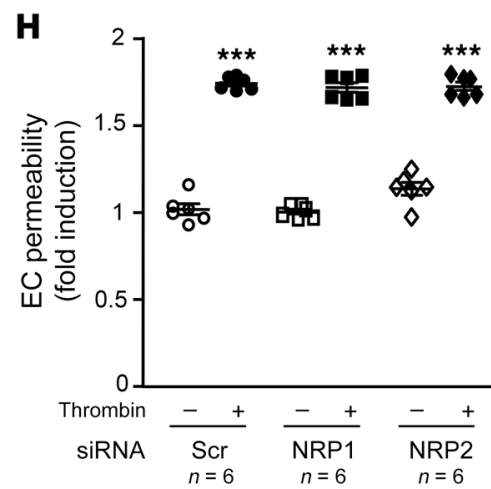

Figure 7. NRP1 and NRP2 bind ANGPTL4 and mediate its promotion of Rho activation and EC permeability. (A-D) Representative sensorgrams of 3 experiments showing binding of VEGF and ANGPTL4 to immobilized NRP1 and NRP2, using SPR binding analysis. KA and KD constants are shown. Ligand concentrations used are $6.25 \mathrm{nM}, 12.5 \mathrm{nM}, 25 \mathrm{nM}$, and $50 \mathrm{nM}$ for binding of VEGFA to NRP1 (A) and NRP2 (B); $3.125 \mathrm{nM}, 6.25 \mathrm{nM}, 12.5 \mathrm{nM}, 25 \mathrm{nM}$, and 50 $\mathrm{nM}$ for binding of ANGPTL4 to NRP1 (C); and $3.125 \mathrm{nM}, 6.25 \mathrm{nM}, 12.5 \mathrm{nM}, 25 \mathrm{nM}$, and $50 \mathrm{nM}$ for binding of ANGPTL4 to NRP2 (D). RU, resonance units. The complete kinetics analysis is listed in Supplemental Table 3. (E) HUVECs were transfected with 50 nM scrambled siRNA, 50 nM NRP1 siRNA, or 50 nM NRP2 siRNA. Inhibition of NRP1 and NRP2 expression is shown. Levels of NRP1, NRP2, and actin were determined in gels run in parallel. (F) Rho activation assay upon transfection of scrambled siRNA, NRP1 siRNA, or NRP2 siRNA and treatment with $5 \mu \mathrm{g} / \mathrm{mL}$ rhANGPTL4 in HUVECs. Positive control: $0.5 \mathrm{U} / \mathrm{mL}$ thrombin. (G and $\mathbf{H}$ ) EC permeability assay upon transfection of scrambled siRNA, NRP1 siRNA, or NRP2 siRNA and treatment with $5 \mu \mathrm{g} / \mathrm{mL}$ rhANGPTL4 (C) or $0.5 \mathrm{U} / \mathrm{mL}$ thrombin $(\mathbf{H})$ in HUVECs. One-way ANOVA $(\mathbf{F}, \mathbf{G}, \mathbf{H}) .{ }^{*} P<0.05$; ${ }^{* *} P<0.01$; ${ }^{* *} P<0.001$. Experiments were repeated at least 3 times.

ated mainly through VEGF receptor 2 (VEGFR2 or the kinase insert domain receptor [KDR]), on vascular ECs (50-52). Thus, we investigated whether treatment of ECs with ANGPTL4, like VEGF, could lead to KDR phosphorylation, a critical event in the signaling through this receptor (52). We observed phosphorylation of KDR in Tyr951 when HUVECs were treated with rhVEGFA, but not when they were treated with rhANGPTL4 (Figure 5D and Supplemental Figure 3). However, the treatment of these cells with both rhANGPTL4 and rhVEGFA resulted in a synergistic increase in phosphorylation in that residue (Figure 5D and Supplemental
Figure 3). Interestingly, treatment of HUVECs with rhANGPTL4 was sufficient to promote phosphorylation of KDR in Tyr1059 (Figure 5D and Supplemental Figure 3). Similar results were observed upon treatment of hRECs with these factors (Figure 5E and Supplemental Figure 4).

We next decided to investigate whether cANGPTL4 could analogously induce the phosphorylation of KDR in treated cells. cANGPTL4 induced an increase in EC permeability, similar to VEGF and full-length ANGPTL4; nANGPTL4 could not reproduce this effect (Figure 5F). cANGPTL4 also promoted destabi- 
A

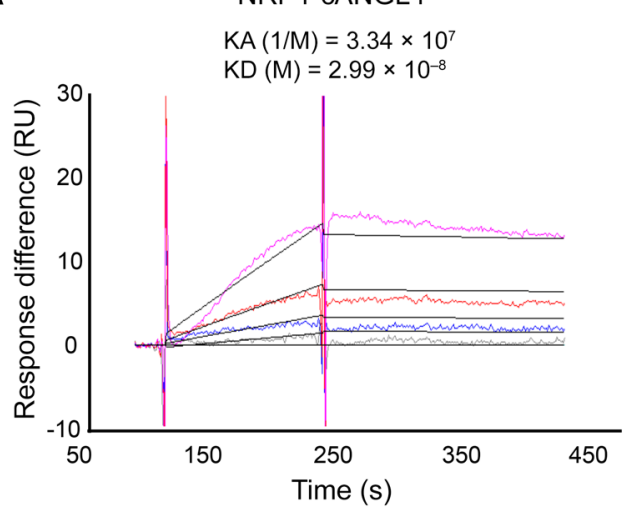

C

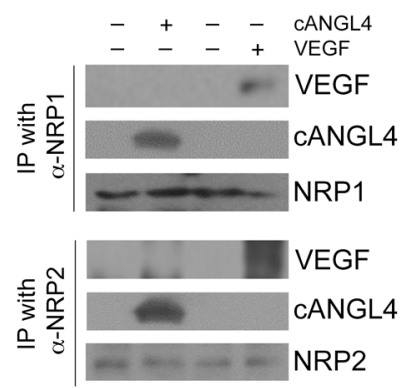

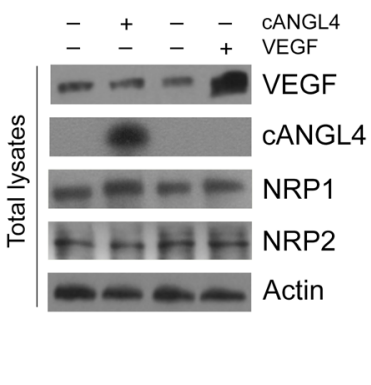

B

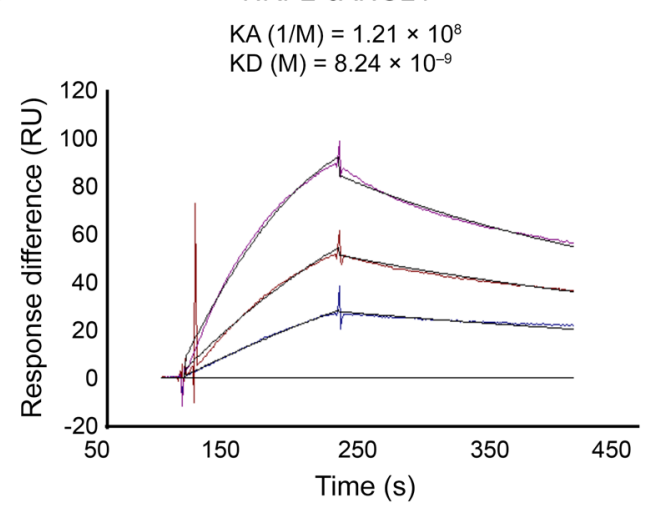

D

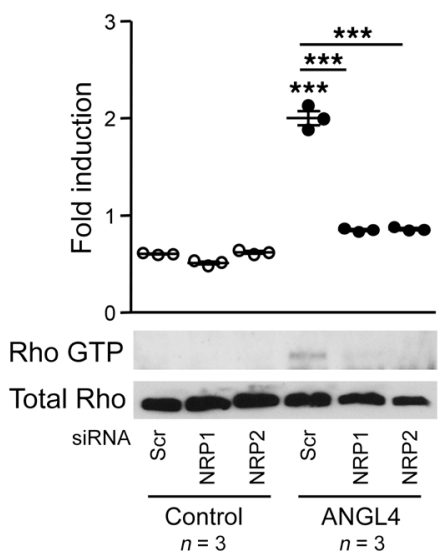

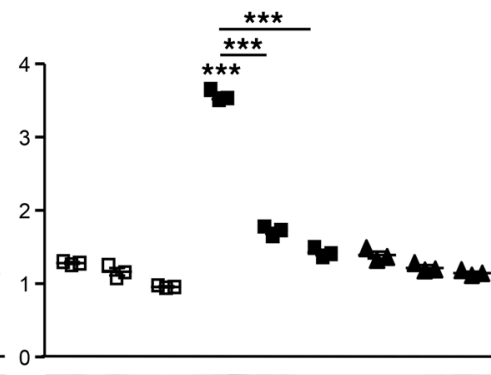

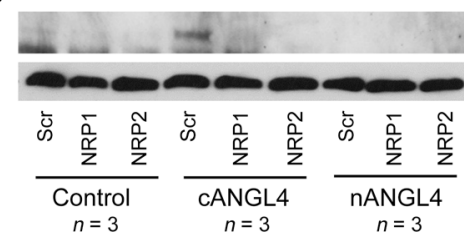

Figure 8. NRP1 and NRP2 bind cANGPTL4 and mediate cANGPTL4-induced promotion of Rho activation. (A and B) Representative sensorgrams of 3 experiments showing binding of cANGPTL4 to immobilized NRP1 and NRP2, using SPR binding analysis. KA and KD constants are shown. The complete kinetics analysis is listed in Supplemental Table 3. cANGPTL4 concentrations are $6.25 \mathrm{nM}, 12.5 \mathrm{nM}, 25 \mathrm{nM}$, and $50 \mathrm{nM}$ for binding of ANGPTL4 to NRP1 (A) and $12.5 \mathrm{nM}, 25 \mathrm{nM}$, and $50 \mathrm{nM}$ for binding of ANGPTL4 to NRP2 (B). (C) Immunoprecipitation of $5 \mu \mathrm{g} / \mathrm{mL}$ rhcANGPTL4 with endogenous NRP1 and NRP2 in HUVECs. Levels of VEGF, CANGPTL4, NRP1, NRP2 and actin were determined in gels run in parallel. (D) Rho assay upon transfection of scrambled siRNA, NRP1 siRNA, or NRP2 siRNA and treatment with $(5 \mathrm{~g} / \mathrm{mL})$ rhANGPTL4, rhcANGPTL4, or rhnANGPTL4 or none (control) in HUVECs. One-way ANOVA (D). ${ }^{* * *} P<0.001$. Experiments were repeated at least 3 times.

lization of the integrity of $\mathrm{AJ}$ and $\mathrm{TJ}$ junctions (Figure $5 \mathrm{G}$ ) and induced KDR phosphorylation on Tyr 951 and Tyr1059 residues (Figure 5H and Supplemental Figure 5). Collectively, these results suggest that ANGPTL4 can signal through KDR and may act synergistically in concert with VEGF in the promotion of retinal vascular permeability.

KDR is not required for ANGPTL 4 promotion of EC permeability. These observations suggested that ANGPTL4 may promote vascular hyperpermeability by usurping effectors downstream from $\mathrm{KDR}$. We therefore next investigated whether KDR was required for the increase in vascular permeability observed following treatment with ANGPTL4. For this purpose, we transfected HUVECs with a specific siRNA to knock down expression of KDR (Figure 6A). Surprisingly, we observed only a marginal reduction of ANGPTL4-mediated promotion of EC permeability by KDR siRNA $(17.6 \% \pm 0.6 \%$ inhibition, which was not statistically significant) (Figure 6B). Similar results were obtained using the pharmacologic inhibitor of KDR, SU1489 (16.9\% $\pm 0.8 \%$ inhibition), and only at the highest tested dose $(1 \mu \mathrm{M})$ of this small molecule inhibitor (Figure 6C). Conversely, knockdown of KDR expression or phar- macologic inhibition of KDR signaling abolished the promotion of EC permeability by VEGF $(94.1 \% \pm 0.2 \%$ inhibition and $99.7 \%$ $\pm 0.7 \%$ respectively) (Figure $6, \mathrm{~B}$ and $\mathrm{C}$ ). We did not observe evidence of binding between ANGPTL4 and KDR using coimmunoprecipitation assays (Figure 6D). Similarly, when we inhibited KDR expression or function in cells treated with rhcANGPTL4, we did not observe a significant role of this VEGFA receptor in the disruption of the EC-EC junctions $(9.4 \% \pm 0.7 \%$ inhibition and 29.1 $\pm 0.4 \%$ inhibition, respectively) (Figure 6, E and F). Collectively, these findings suggest that, although ANGPTL4 and VEGFA cooperate in the induction of vascular hyperpermeability and despite our observation that ANGPTL4 promotes KDR phosphorylation, this VEGFA receptor appears to facilitate, but is not strictly required for, ANGPTL4 to promote vascular leakage.

Neuropilin 1 and 2 bind ANGPTL4 and are necessary for ANGPTL4 to promote Rho activation and EC permeability. ANGPTL4 has previously been reported to bind a handful of membrane proteins, including $\beta_{1}, \beta_{3}$, and $\beta_{5}$ integrins $(23,53,54)$. Nonetheless, ANGPTL4 (and other members of the ANGPTL family) are considered orphan ligands. The observation that KDR 
A

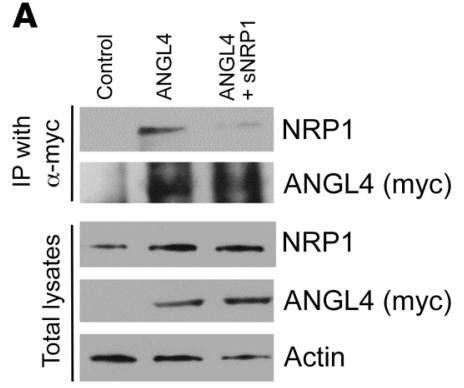

B

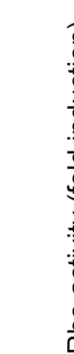

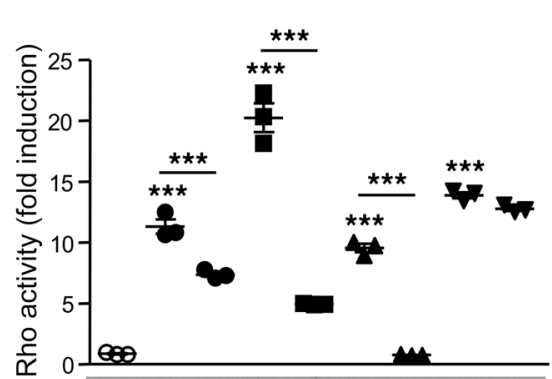

Active Rho

Total Rho

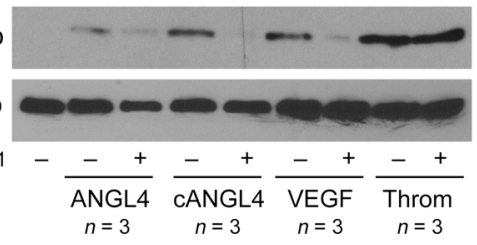

C

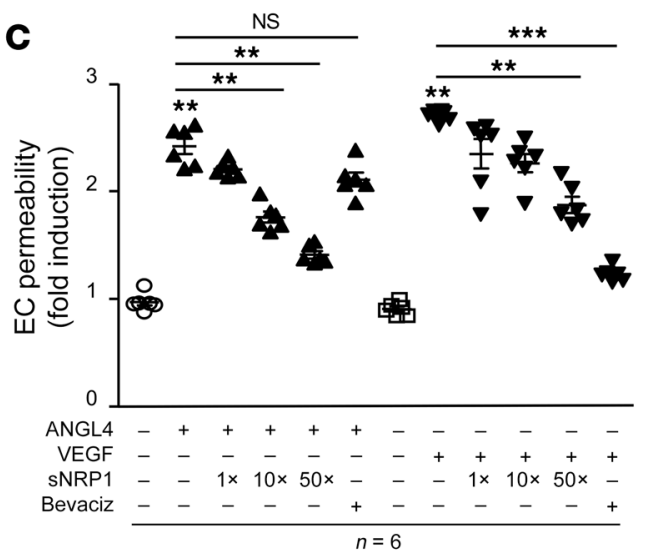

D

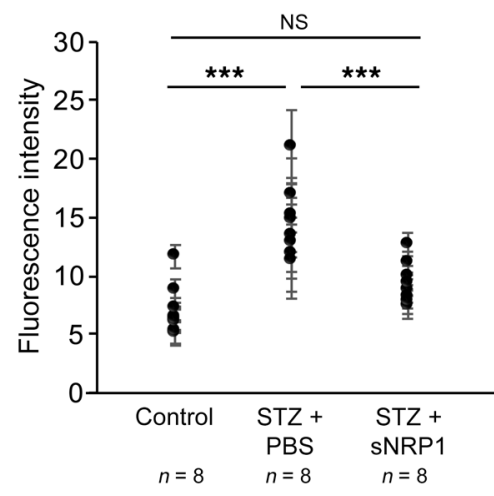

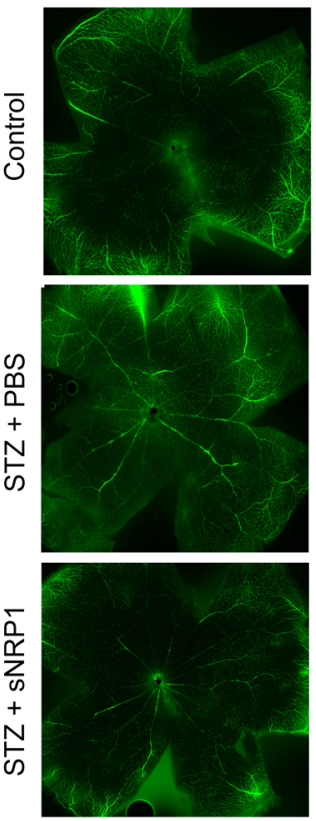

E

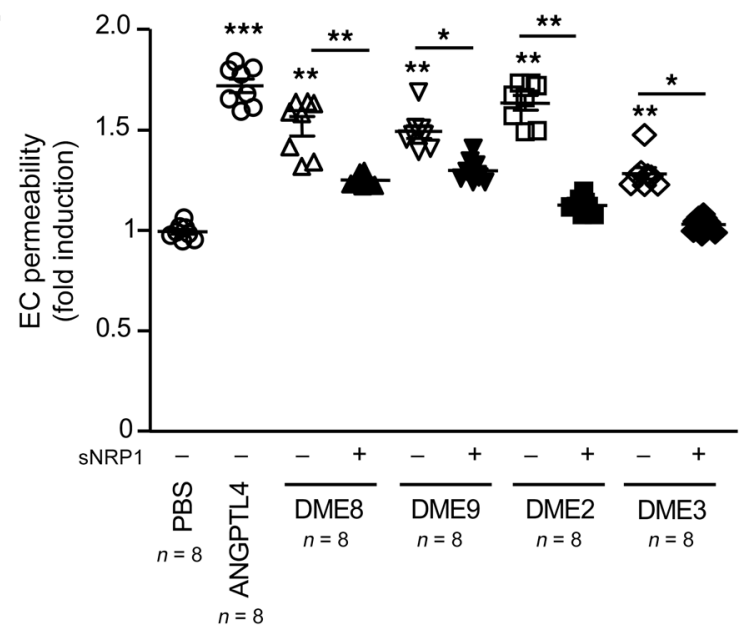

Figure 9. sNRP1 blocks ANGPTL4 activation of RhoA and vascular permeability in vitro and in vivo. (A) Immunoprecipitation of ANGPTL4 with endogenous NRP1 upon transfection of pcDNA3.1-ANGPTL4-mycHis and addition or not of $113 \mathrm{nM}$ sNRP1 in HUVECs. (B) Rho assay upon treatment with $5 \mu \mathrm{g} /$ $\mathrm{mL}$ rhANGPTL4 (ANGL4), $5 \mu \mathrm{g} / \mathrm{mL}$ rhcANGPTL4 (cANGL4), $50 \mathrm{ng} / \mathrm{mL}$ rhVEGF (VEGF), or $0.5 \mathrm{U} / \mathrm{mL}$ thrombin (Throm) in the presence or absence of sNRP1 in HUVECs. sNRP1 concentration used was $113 \mathrm{nM}$ (for rhANGPTL4 or rhcANGPTL4), $178 \mathrm{nM}$ (for thrombin), and $1.3 \mathrm{nM}$ (for VEGF). (C) EC permeability upon treatment of HUVECs with $5 \mu \mathrm{g} / \mathrm{mL}$ rhANGPTL4 or $50 \mathrm{ng} / \mathrm{mL}$ rhVEGF along with $1 \times$, 10x, or $50 \times$ sNRP1 ( $x=113 \mathrm{nM}$ for ANGPTL4; $x=1.3 \mathrm{nM}$ for VEGF) or 100 $\mu \mathrm{g} / \mathrm{mL}$ vevacizumab. (D) Quantification of retinal vascular permeability and immunofluorescence of corresponding flat retinas in 8 -month-old nondiabetic (control) and diabetic STZ mice $(n=8)$ upon intraretinal injection of PBS or sNRP1 (200 ng/ $\mu \mathrm{L})$. Original magnification, $\times 5$. (E) EC permeability upon treatment of aqueous samples from DME patients (DME8, DME9, DME2, DME3; Supplemental Table 1) with $10 \mathrm{nM}$ sNRP1. One-way ANOVA (B-E). * $P<0.05$; ${ }^{* *} P<0.01 ;{ }^{* *} P<0.001$. Experiments were repeated at least 3 times.

is not required for the promotion of vascular hyperpermeability by ANGPTL4 suggests that another EC receptor may be responsible for this function. Yet the synergistic effects of ANGPTL4 with VEGF support the possibility of a role for a shared receptor between these two factors.

Based on these observations, we hypothesized that the receptor that mediates ANGPTL4 signaling may be a coreceptor that regulates vascular hyperpermeability by VEGFA (and/or KDR). In this regard, the neuropilin (NRP) receptors are transmembrane glycoproteins with an essential role in the control of angiogenesis that have been reported to bind VEGFs and regulate their function $(55,56)$. The formation of complexes containing KDR and NRPs has been shown to increase VEGF binding to KDR and to regulate VEGF-mediated angiogenesis and endothelial permeability (57, 58). Indeed, while binding of VEGF to KDR moderately activates its intracellular kinase activity, NRPs are required for strong and sustained kinase activation (57). There are 2 conserved NRP family members in vertebrates, NRP1 and NRP2, which share the same overall domain structure and are $44 \%$ identical at the amino acid level. Ligands for NRP1 and NRP2 have been shown to bind sulfate 


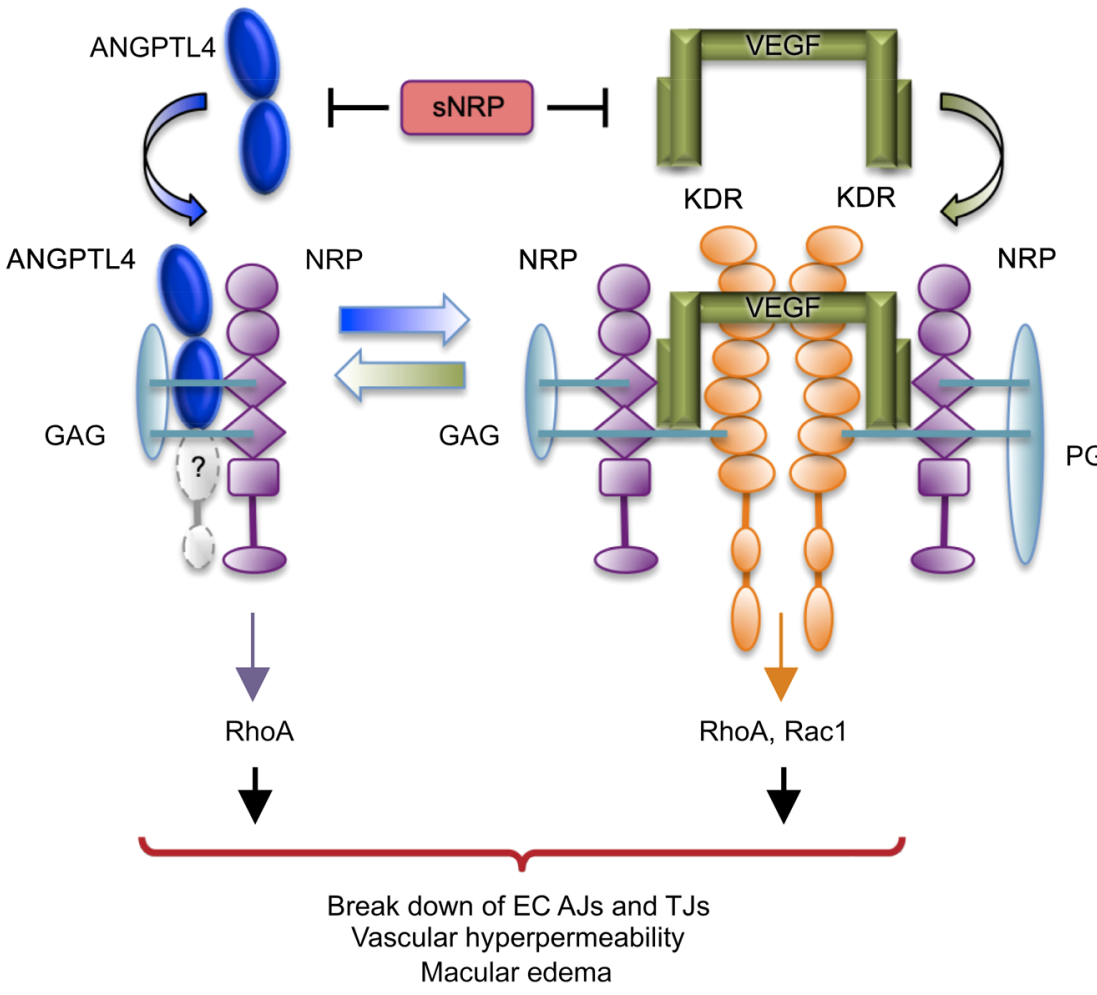

Figure 10. Schematic showing the binding of ANGPTL4 to NRP as well as the binding of VEGF to KDR and NRP. Glycosaminoglycans (GAG) and proteoglycans (PG) have also shown to interact with these ligands and receptors in the plasma membrane. An sNRP would inhibit both ANGPTL4 and VEGF.

proteoglycans and/or undergo processing by cleavage by furin or furin-like convertases (59-61). Interestingly, ANGPTL4 has been reported to bind sulfate proteoglycans and undergo processing by cleavage by furin (62).

We therefore set out to determine whether NRPs could provide the link among ANGPTL4, VEGF, and the regulation of vascular EC-EC junctions. To this end, we performed studies using surface plasmon resonance (SPR) to investigate the possible interaction between NRP1 and NRP2 and ANGPTL4. As previously reported, VEGF demonstrated strong binding to NRP1 and NRP2 with dissociation constants of $6.65 \times 10^{-9} \mathrm{M}$ and $6.18 \times 10^{-9} \mathrm{M}$, respectively (Figure 7, A and B, and Supplemental Table 3). Interestingly, we observed ANGPTL4 also strongly bound immobilized NRP1 and NRP2 with dissociation constants of $2.50 \times 10^{-9} \mathrm{M}$ and $7.07 \times 10^{-9}$ M, respectively (Figure 7, C and D, and Supplemental Table 3).

To elucidate whether NRP1 and NRP2 mediate the signal transduction pathways induced upon treatment of ECs with ANGPTL4, we blocked NRP1/2 expression with specific siRNAs in HUVECs (Figure 7E). We observed inhibition of the induction of RhoA activation (Figure 7F) as well as an inhibition of the induction of EC permeability by ANGPTL4 (Figure 7G) upon downregulation of NRP1 or NRP2 expression. Conversely, the induction of EC permeability by thrombin, which does not bind to NRPs, was not affected by the knockdown of NRP1 or NRP2 (Figure 7H). Collectively, these results suggest that ANGPTL4 binds to NRP1 and NRP2 and that binding to NRPs is important for the RhoA-dependent destabilization of the EC-EC barrier triggered by this hyperpermeability factor.
NRP1 and NRP2 bind cANGPTL4 and mediate its promotion of Rho activation. We next performed SPR studies to examine the possible interaction between NRP1 and NRP2 and cANGPTL4. We observed strong binding of cANGPTL4 to NRP1 and NRP2 (Figure 8, A and $\mathrm{B})$ with similar dissociation constants $\left(\mathrm{K}_{\mathrm{D}}\right.$ $=2.99 \times 10^{-8} \mathrm{M}$ and $\mathrm{K}_{\mathrm{D}}=8.24 \times 10^{-9} \mathrm{M}$, respectively) to VEGF (Figure 8, A and B, and Supplemental Table 3). To corroborate these SPR data in ECs, we performed immunoprecipitation assays in HUVECs following treatment with rhcANGPTL4 and found cANGPTL4 in immunocomplexes along with the NRP1 and NRP2 receptors (Figure 8C). Accordingly, we observed that cANGPTL4, but not nANGPTL4, promotes the activation of RhoA GTPase, an effect that was blocked by the knockdown of NRP1 or NRP2 expression using siRNA (Figure 8D). Collectively, these results suggest that NRP1 and NRP2 are important receptors mediating the vasoactive effects of cANGPTL4.

ANGPTL4 signaling is blocked by soluble NRP1. Soluble NRPs (sNRPs) are naturally occurring fragments of NRPs that lack the transmembrane and intracellular domains. They are expressed independently of intact NRPs and function as endogenous inhibitors of the biological effects of NRP signaling by acting as traps for the NRP ligands (55). We therefore next set out to determine whether these endogenous NRP inhibitors could provide an effective therapeutic approach to block the effects of ANGPTL4 on vascular hyperpermeability. Treatment of HUVECs with exogenous SNRP1 prevented binding of endogenous NRP1 to ANGPTL4 (Figure 9A). Accordingly, we observed a potent inhibition of ANGPTL4-induced RhoA activation and EC permeability in HUVECs treated with sNRP1 (Figure 9, B and C).

We next examined the effect of treating STZ mice with sNRP1. Figure 9D shows that intraocular injection of rhsNRP1 was able to block the promotion of retinal vascular leakage in diabetic animals compared with the animals treated with vehicle (PBS) control. To examine the therapeutic potential of treating patients with exogenous sNRP1, we next investigated the ability of this endogenous antivascular hyperpermeability protein to inhibit EC permeability induced by the aqueous fluid from patients with DME. Interestingly, we observed a marked decrease in EC permeability induced by the DME samples in cells pretreated with sNRP1 (Figure 9E). Collectively, these results suggest that sequestering both ANGPTL4 and VEGF using sNRP1 could be an effective therapeutic approach for the treatment of DME (Figure 10).

\section{Discussion}

Therapies targeting VEGF have had a remarkable impact on the treatment of patients with DME (44). However, several multicenter randomized, controlled clinical trials have demonstrated that antiVEGF therapies result in a clinically significant improvement in vision in only a subset of these patients $(45,63)$. We have previously 
demonstrated that the expression of a vasoactive factor, ANGPTL4, is increased in the ischemic inner retina in hypoxic Müller glial cells (24). We observed that ANGPTL4 upregulation leads to the promotion of vascular hyperpermeability and retinal neovascularization in patients with ischemic retinal disease $(24,38)$.

Of note, it has been previously reported that plasma levels of ANGPTL4 are increased in patients with the metabolic syndrome and type 2 diabetes (64) and that ANGPTL4, in turn, may promote retinal inflammation in diabetic patients by activating Profilin-1 (65). These results suggest that ANGPTL4 expression may be observed earlier in diabetic eye disease as a consequence of early inflammatory changes, but prior to the development of retinal ischemia. We demonstrate here that ANGPTL4 expression is increased in the eyes of hyperglycemic mice and in the aqueous fluid of diabetic patients with nonischemic, nonproliferative diabetic retinopathy, supporting a role for this cytokine in early diabetic eye disease.

Much of our knowledge about ANGPTL4 function derives from the important role of its $\mathrm{N}$-terminal domain in regulating lipid metabolism, glucose homeostasis, and insulin sensitivity $(9,11)$. However, the role of cANGPTL4 in the promotion of angiogenesis and vessel permeability has been more controversial. Preclinical data supporting a role for ANGPTL4 in suppressing angiogenesis and vessel permeability $(62,66-68)$ and preserving vascular integrity $(69,70)$ have been reported. These conflicting studies may be a consequence of diverse experimental approaches and disease-specific animal models as well as context- and tissue-specific activities for ANGPTL4 (5). Posttranslational modifications and proteolytic processing of ANGPTL4 may differ in these settings and may influence its biological roles $(7,62)$. Elucidation of the structural details that help determine the different functions of this unique factor may ultimately provide an explanation for these discordant observations.

Of particular interest is the observation that ANGPTL4 may promote - rather than disrupt - vessel integrity during retinal development (71). However, these seemingly paradoxical functions for ANGPTL4 are not uncommon. Many factors that control cell behavior can play different roles depending on different physiologic/pathologic conditions. The most appropriate example may be VEGF, which is essential in vascular development, yet it is clearly an important pathologic mediator of dysregulated vascular proliferation and leakage $(72,73)$. The data on vessel maturation and stability described in ANGPTL4-deficient mice are therefore not in opposition to the accumulating evidence that supports a role for ANGPTL4 in the promotion of pathological angiogenesis and vascular hyperpermeability in several biological circumstances $(22,23,25-27,31,33,34,37,74-78)$.

Our results reported here on the role of ANGPTL4 in diabetic eye disease are consistent with prior reports showing that aqueous fluid levels of ANGPTL4 correlate with nonperfusion and macular volume in diabetic retinopathy as well as the observation that expression of this cytokine is markedly elevated in the vitreous of patients with diabetic eye disease $(24,38,45,77,79,80)$. It has also been reported that aqueous ANGPTL4 levels correlate with nonperfusion area and ME in branch retinal vein and artery occlusions $(40,81)$. And an association between ANGPTL4 and pathological angiogenesis has further been found in age-related macular degeneration (82), sickle retinopathy (39), pterygia (83), and uveal melanoma (39). The emerging evidence implicating ANGPTL4 in the pathogenesis of a growing list of ocular neovascular diseases suggests that this factor may have broad influence on the development of many vision-threatening diseases.

Here, we show that ANGPTL4-induced disruption of the integrity of the EC monolayer is mediated by the rapid activation of RhoA/Rho kinase, leading to actomyosin contraction and loss of EC-EC adhesion. Many hyperpermeability factors, including thrombin, histamine, bradykinin, and platelet-activating factor (PAF) promote vascular leakage by activation of RhoA (48). Rho kinases (ROCK1 and ROCK2) and PKC-related kinase (PRK), all RhoA downstream effectors, are pivotal proteins in the contraction of actomyosin fibers and in the loss of cell-cell attachment by regulation of MLC phosphorylation (49). By regulating the actin cytoskeletal assembly, ROCKs play an important role, not only in EC permeability, but also in macrophage phagocytic activity, cell migration, cellular metabolism, cellular growth, and apoptosis. ROCK inhibitors are being considered for the therapeutic management of pulmonary hypertension as well as other diseases, including asthma, cancer, kidney failure, neuronal degeneration, glaucoma, and osteoporosis (84-89). In the eye, ROCK inhibitors have been suggested to block retinal pigment epithelium (RPE) dysfunction, microvascular damage, and cell death caused by ischemia/reperfusion injury in ischemic retinopathies (90-92). Our results suggest that the Rho/ROCK pathway could be an effective target also for the treatment of DME.

One of the obstacles to understanding ANGPTL4 signaling has been the lack of known receptors for this ligand. In this regard, we report here that treatment of ECs with ANGPTL4 causes phosphorylation of the VEGF receptor, KDR. However, despite evidence that VEGF and ANGPTL4 activation of KDR phosphorylation may be additive, we could not find direct binding of ANGPTL4 with KDR. Moreover, inhibition of KDR expression or signaling only had a modest impact on ANGPTL4-induced EC-EC hyperpermeability, suggesting that ANGPTL4 also signals into the cell independently of KDR. Nonetheless, our observation that ANGPTL4 promotes KDR phosphorylation and can enhance the promotion of KDR phosphorylation by VEGF, but that it does not bind directly to KDR, collectively suggest that the binding of ANGPTL4 to its membrane receptors may influence KDR and/or VEGF/KDR interaction. This suggests that therapies targeting ANGTL4 may influence VEGF/ KDR signaling and has important implications for the development of therapies targeting the VEGF/KDR axis.

We further provide evidence supporting a role for NRP1 and NRP2 as the EC receptors that are directly responsible for the promotion of vascular hyperpermeability by ANGPTL4. We demonstrate that ANGPTL4 binds both NRP1 and NRP2 with comparable affinities to VEGFA/NRPs binding. NRPs are essential cell-surface proteins that play pivotal functions in different tissues and organs, including the vascular and lymphatic system $(55,56)$. Besides binding VEGFs, they are coreceptors of Semaphorin3s and play a role in axon guidance, and they have also shown to interact with other factors, including fibroblast growth factor, platelet-derived growth factor, hepatocyte growth factor, and transforming growth factor- $\beta 1(55,56)$. Interestingly, the phenotype of NRP1 knockout mice demonstrates embryonic lethality due to widely distributed defects in vasculogenesis and vascular patterning, while NRP2 has 
been shown to be important for VEGFC/VEGFR2/R3-mediated lymphangiogenesis (93-95). Targeting of both NRP1 and NRP2 genes severely impairs embryonic angiogenesis, demonstrating a critical function of these transmembrane proteins in the developing vasculature (96). Here, we report that NRP1 and NRP2 are essential for the signaling of ANGPTL4 to RhoA-ROCK kinase and the promotion of vascular leakage, suggesting that the ANGPTL4/ NRP axis may be important in the pathogenesis of DME.

It has previously been reported that the neuronal guidance cue semaphorin 3A may also promote vascular permeability and, in turn, DME via its cognate receptor NRP1 (97). Interestingly, the authors reported that expression of semaphorin $3 \mathrm{~A}$ is observed earlier in diabetic animals than is that of VEGF, suggesting that neutralization of semaphorin 3A may more efficiently prevent retinal vascular leakage in early stages of diabetic eye disease, prior to the development of retinal ischemia, when VEGF expression is not yet observed. We similarly report robust expression of ANGPTL4 in diabetic animals at a stage when increased VEGF expression is not yet observed. Taken together, these results suggest that NRP1 may participate in the breakdown of endothelial barrier function through different stages of diabetic eye disease and that therapies targeting this receptor may be an effective approach for targeting all 3 vascular hyperpermeability factors at all stages of this disease.

Collectively, our studies suggest that combined anti-VEGF and anti-ANGPTL4 therapies could be more advantageous to therapies targeting VEGF (or ANGPTL4) alone. In this regard, we demonstrate here that SNRP1, the endogenous competitive inhibitor of NRP signaling, blocks ANGPTL4 binding to NRP1, inhibits ANGPTL4 from signaling to RhoA, and prevents the promotion of EC permeability by this hyperpermeability factor. Indeed, we see a reduction of ANGPTL4 vessel permeability by sNRP1 in vivo as well as in diabetic mice and in samples from DME patients. Similarly, deficiencies in NRPs have shown to suppress VEGF-induced retinal neovascularization (98), and a recombinant mouse sNRP1 has been used as a bivalent trap to neutralize semaphorin $3 \mathrm{~A}$ and VEGF in STZ-treated mice (97). It is tempting to speculate that the promising therapeutic potential of sNRP1 as an approach for the treatment of DME relies on its ability to block multiple pathogenic vasoactive molecules.

\section{Methods}

Cell culture and reagents. Immortalized HUVECs were obtained from J. Silvio Gutkind (UCSD, La Jolla, California, USA) and cultured as previously described (99). Primary hRECs were purchased from Cell Systems. Recombinant human and murine (full length) ANGPTL4 (rhANGPTL4, rmANGPTL4), human C-terminal domain ANGPTL4 (rhcANGPTL4), human N-terminal domain ANGPTL4 (rhnANGPTL4), and human or murine VEGFA (rhVEGF, rmVEGF) were purchased from R\&D Systems. pcDNA3.1-ANGPTL4-mycHis (full-length human ANGPTL4) was provided by Stéphane Germain (Centre for Interdisciplinary Research in Biology, Paris, France). ANGPTL4 and VEGF ELISA kits were obtained from R\&D Systems as well. SU1498, HA1077, blebbistatin, and ML7 inhibitors were obtained from Calbiochem. C3 toxin was purchased from List Biological Laboratories. The RhoA, Rac1, and Cdc42 activity assays were purchased from Cell Biolabs. Bevacizumab was obtained from the Johns Hopkins University Pharmacy. Predesigned control (scrambled), KDR, NRP1, and NRP2 siRNA sequences were obtained from QIAGEN. siRNA delivery to cells was performed using Hiperfect (QIAGEN).

$S P R$ assays. SPR binding assays were performed at the UMB Biacore Facility to investigate the interactions in vitro of human ANGPTL4, cANGPTL4, nANGPTL4, or VEGF (R\&D Systems) with the human NRP1-Fc protein or NRP2-Fc protein (R\&D Systems), using Biacore T3000 equipment. NRP1-Fc and NRP2-Fc were immobilized directly onto a CM5 sensor chip (catalog BR1000-12, GE Health Science) via amine coupling (catalog BR-1000-50, GE Health Science), as described previously (100). The running buffer and sample buffer were HBS-EP containing $10 \mathrm{mM}$ HEPES, $150 \mathrm{mM} \mathrm{NaCl}, 3 \mathrm{mM}$ EDTA, 0.05\% P20 (catalog BR-1006-70, GE Health Science). A control flow cell was prepared by omitting NRP1 or NRP2. Several concentrations of ANGPTL4s (3.125 nM, $6.25 \mathrm{nM}, 12.5 \mathrm{nM}, 25 \mathrm{nM}, 50 \mathrm{nM}, 100 \mathrm{nM}$ ) were injected over immobilized NRPs at $30 \mu \mathrm{l} / \mathrm{min}$ and $25^{\circ} \mathrm{C}$. The association (ka) and dissociation (kd) rate constants and the equilibrium association (KA) and dissociation (KD) constants were calculated with the BIAeval 3.2 evaluation software.

Western blot, immunoprecipitation, immunofluorescence and ELISA. Cell lysates and immunocomplexes were subjected to $4 \%-15 \%$ gradient SDS/PAGE (Invitrogen). Immunoblot assays were performed with primary antibodies specifically recognizing ZO1 (Invitrogen, catalog 402200), ZO2 (Invitrogen, catalog 711400), ZO3 (Invitrogen, catalog 364100), claudin 5 (Invitrogen, catalog 352500), occludin (Invitrogen, catalog 331500), VE-cadherin (Santa Cruz Biotechnology Inc., catalog sc-9989), $\beta$-catenin (Santa Cruz Biotechnology Inc., catalog sc-7936), actin (Proteintech, catalog 60008), p-KDR ${ }^{\mathrm{Y} 91}$ (Cell Signaling Technology, catalog 4991), p-KDR ${ }^{\mathrm{Y} 1059}$ (Cell Signaling Technology, catalog 3817), KDR (Cell Signaling Technology, catalog 2479), myc (Cell Signaling Technology, catalog 2276) VEGFA (Proteintech, catalog 19003-1-AP), ANGPTL4 (Proteintech, catalog 18374-1-AP), anti-mouse HRP secondary antibody (Bio-Rad, catalog 1706516), and anti-rabbit HRP secondary antibody (Bio-Rad, catalog 1706515). Rho GTPase antibodies were provided in the RhoA, Rac1, and Cdc42 activity assay kits. Levels of secreted VEGF and ANGPTL4 were measured in conditioned media using DuoSet human VEGF or ANGPTL4 ELISA kits (R\&D Systems). Immunofluorescence images were captured with Cytation 5 (BioTek), using the aforementioned antibodies against $\mathrm{ZO} 1$ and $\beta$-catenin, Alexa Fluor 594 anti-rabbit antibody (Invitrogen, catalog A11012), and Alexa Fluor 594 anti-mouse antibody (Invitrogen, catalog A11032).

EC permeability assays. In vitro EC permeability assay were performed as previously described (21). Briefly, HUVECs were seeded on collagen-coated Transwells ( $3 \mathrm{~mm}$ size pore; PTFE, Corning) and allowed to grow as a 3-day-old mature monolayers. After overnight starvation, 500 and $100 \mu \mathrm{L}$ media with corresponding treatments were added for 30 minutes $\left(37^{\circ} \mathrm{C}\right)$ to the bottom and top chambers, respectively. A total of $100 \mu \mathrm{L} 1 \mathrm{mg} / \mathrm{mL}$ FITC-dextran (molecular weight = 40,000 daltons; Invitrogen) was added for 30 minutes. Fluorescence was quantified using a SpectraMax M5 Microplate Reader (Molecular Devices) with excitation at $494 \mathrm{~nm}$ and emission at $521 \mathrm{~nm}$.

STZ mouse model. Seven- to eight-week-old C57BL/6J mice (The Jackson Laboratory) were weighed and their glycemia levels were quantified. Mice were injected intraperitoneally with STZ (SigmaAldrich) for 5 consecutive days at $55 \mathrm{mg} / \mathrm{kg}$. Age-matched controls were injected with buffer. Glycemia was measured again a week after the last STZ injection. Mice were considered diabetic when their nonfasted glycemia was higher than $17 \mathrm{mM}$ (300 mg/dL). 
Intraocular injections. Intraocular injections of C57BL/6J mice with $1 \mu \mathrm{L}$ of rmVEGF (100 ng/ $\mu \mathrm{L})$ or rmANGPTL4 (200 ng/ $\mu \mathrm{L})$ were performed with a nanofil syringe (World Precision Instruments) using a 36-gauge beveled needle (24).

Human samples. Inclusion criteria for patients with DME included a known diagnosis of diabetes and the presence of ME (observed on clinical exam and spectral domain optical coherence tomography). Exclusion criteria for control patients included diabetes or any known ischemic retinal disease. Ocular samples were collected from consenting patients at the Wilmer Eye Institute undergoing intravitreal injection (aqueous fluid samples for diabetic eyes) or cataract surgery (aqueous fluid samples for control eyes). Aqueous fluid samples were obtained immediately after performing intravitreal injection or immediately prior to initiating cataract surgery (using a 30-gauge needle). Control samples were obtained from patients without a history of ischemic retinal disease at the time of surgery. Exclusion criteria included any other ischemic retinal disease, uveitis, retinal detachment within 1 year of sample collection, or neovascularization from another cause. Aqueous samples were immediately processed and stored at $-80^{\circ} \mathrm{C}$ prior to analysis.

Study approval. All mice in the study were treated in accordance with the Association for Research in Vision and Ophthalmology Statement for the Use of Animals in Ophthalmic and Vision Research and the guidelines of the Johns Hopkins University Animal Care and Use Committee. Institutional Review Board approval from the Johns Hopkins University School of Medicine was obtained for all patient samples used in this study. Written informed consent was received from participants prior to inclusion in the study. Consent was written and voluntary without stipend.

Statistics. In all cases, results are shown as a mean value \pm SD from at least 3 independent experiments. Western blot scans are represen- tative of at least 3 independent experiments. Statistical differences between groups were determined using 2-tailed unpaired Student's $t$ test, Mann-Whitney $U$ test, or 1-way ANOVA with post hoc Tukey's honestly significant difference test. Correlation was tested using Pearson's method. Statistical analysis was performed using Microsoft Office and Prism 6.0 software (Graph-Pad).

\section{Author contributions}

SM and AS designed the research. TM, MD, DM, KJ, AD, JD, and DL performed the research. SM, AS, TM, MD, DM, KJ, AD, JD, and DL analyzed the data. SM and AS wrote the paper.

\section{Acknowledgments}

This work was supported by NIH grant 5R01EY025705 to SM and AS. AS is also supported by an unrestricted grant from Research to Prevent Blindness. AS gratefully acknowledges the support he receives as a Special Scholar Award recipient from Research to Prevent Blindness, Inc. and from the Branna and Irving Sisenwein Professorship in Ophthalmology. We thank Robert Bloch and Yinghua Zhang (UMB Biosensor/Biacore Shared Facility) for their help with the SPR experiments.

Address correspondence to: Silvia Montaner, Department of Oncology and Diagnostic Sciences, University of Maryland, 650 W. Baltimore Street, 7th North, Room 7263, Baltimore, Maryland 21201, USA. Phone: 410.706.7944; Email: smontaner@ umaryland.edu. Or to: Akrit Sodhi, Wilmer Eye Institute, Johns Hopkins School of Medicine, 400 N. Broadway Street, Smith Building, Room 4039, Baltimore, Maryland 21287, USA. Phone: 410.614.6682; Email: asodhi1@jhmi.edu.
1. Hato T, Tabata M, Oike Y. The role of angiopoietin-like proteins in angiogenesis and metabolism. Trends Cardiovasc Med. 2008;18(1):6-14.

2. Santulli G. Angiopoietin-like proteins: a comprehensive look. Front Endocrinol (Lausanne). 2014;5:4.

3. Quagliarini F, et al. Atypical angiopoietin-like protein that regulates ANGPTL3. Proc Natl Acad Sci U S A. 2012;109(48):19751-19756.

4. Tan MJ, Teo Z, Sng MK, Zhu P, Tan NS. Emerging roles of angiopoietin-like 4 in human cancer. $\mathrm{Mol}$ Cancer Res. 2012;10(6):677-688.

5. Zhu P, Goh YY, Chin HF, Kersten S, Tan NS. Angiopoietin-like 4: a decade of research. Biosci Rep. 2012;32(3):211-219.

6. Yin W, Romeo S, Chang S, Grishin NV, Hobbs $\mathrm{HH}$, Cohen JC. Genetic variation in ANGPTL4 provides insights into protein processing and function. J Biol Chem. 2009;284(19):13213-13222.

7. Lei X, et al. Proteolytic processing of angiopoietin-like protein 4 by proprotein convertases modulates its inhibitory effects on lipoprotein lipase activity. J Biol Chem. 2011;286(18):15747-15756.

8. Zhang R. The ANGPTL3-4-8 model, a molecular mechanism for triglyceride trafficking. Open Biol. 2016;6(4):150272.

9. Dijk W, Kersten S. Regulation of lipid metabolism by angiopoietin-like proteins. Curr Opin Lipidol. 2016;27(3):249-256.

10. Dewey FE, et al. Inactivating variants in
ANGPTL4 and risk of coronary artery disease. N Engl J Med. 2016;374(12):1123-1133.

11. Kadomatsu T, Tabata M, Oike Y. Angiopoietinlike proteins: emerging targets for treatment of obesity and related metabolic diseases. FEBS J. 2011;278(4):559-564.

12. Gusarova V, et al. Genetic inactivation of ANGPTL4 improves glucose homeostasis and is associated with reduced risk of diabetes. Nat Commun. 2018;9(1):2252.

13. Kim I, et al. Molecular cloning, expression, and characterization of angiopoietin-related protein. Angiopoietin-related protein induces endothelial cell sprouting. J Biol Chem. 1999;274(37):26523-26528.

14. Watanabe D, et al. Vitreous levels of angiopoietin 2 and vascular endothelial growth factor in patients with proliferative diabetic retinopathy. Am JOphthalmol. 2005;139(3):476-481.

15. Das A, Fanslow W, Cerretti D, Warren E, Talarico N, McGuire P. Angiopoietin/Tek interactions regulate mmp-9 expression and retinal neovascularization. Lab Invest. 2003;83(11):1637-1645.

16. Pfister F, et al. Pericyte migration: a novel mechanism of pericyte loss in experimental diabetic retinopathy. Diabetes. 2008;57(9):2495-2502.

17. Feng Y, et al. Impaired pericyte recruitment and abnormal retinal angiogenesis as a result of angiopoietin-2 overexpression. Thromb Haemost. 2007;97(1):99-108.
18. Yao D, et al. High glucose increases angiopoietin-2 transcription in microvascular endothelial cells through methylglyoxal modification of mSin3A. J Biol Chem. 2007;282(42):31038-31045.

19. Ohashi $\mathrm{H}$, et al. Alterations in expression of angiopoietins and the Tie- 2 receptor in the retina of streptozotocin induced diabetic rats. Mol Vis. 2004;10:608-617.

20. Rangasamy S, Srinivasan R, Maestas J, McGuire PG, Das A. A potential role for angiopoietin 2 in the regulation of the blood-retinal barrier in diabetic retinopathy. Invest Ophthalmol Vis Sci. 2011;52(6):3784-3791.

21. Ma T, et al. Viral G protein-coupled receptor up-regulates Angiopoietin-like 4 promoting angiogenesis and vascular permeability in Kaposi's sarcoma. Proc Natl Acad Sci U S A. 2010;107(32):14363-14368.

22. Padua D, et al. TGFbeta primes breast tumors for lung metastasis seeding through angiopoietin-like 4. Cell. 2008;133(1):66-77.

23. Huang RL, et al. ANGPTL4 modulates vascular junction integrity by integrin signaling and disruption of intercellular VE-cadherin and claudin-5 clusters. Blood. 2011;118(14):3990-4002.

24. Xin X, et al. Hypoxic retinal Muller cells promote vascular permeability by HIF-1-dependent up-regulation of angiopoietin-like 4. Proc Natl Acad Sci U S A. 2013;110(36):E3425-E3434.

25. Nakayama T, et al. Expression of angiopoietin-like 
4 (ANGPTL4) in human colorectal cancer: ANGPTL4 promotes venous invasion and distant metastasis. Oncol Rep. 2011;25(4):929-935.

26. Nakayama T, Hirakawa H, Shibata K, Abe $\mathrm{K}$, Nagayasu T, Taguchi T. Expression of angiopoietin-like 4 in human gastric cancer: ANGPTL4 promotes venous invasion. Oncol Rep. 2010;24(3):599-606.

27. Zhang H, et al. HIF-1-dependent expression of angiopoietin-like 4 and L1CAM mediates vascular metastasis of hypoxic breast cancer cells to the lungs. Oncogene. 2012;31(14):1757-1770.

28. Hata S, et al. Hypoxia-induced angiopoietin-like protein 4 as a clinical biomarker and treatment target for human prostate cancer. Oncol Rep. 2017;38(1):120-128.

29. Ng KT, et al. Clinical relevance and therapeutic potential of angiopoietin-like protein 4 in hepatocellular carcinoma. Mol Cancer. 2014;13:196.

30. El-Shal AS, Zidan HE, Rashad NM, Wadea FM. Angiopoietin-like protein 3 and 4 expression 4 and their serum levels in hepatocellular carcinoma. Cytokine. 2017;96:75-86.

31. Tanaka J, et al. ANGPTL4 regulates the metastatic potential of oral squamous cell carcinoma. J Oral Pathol Med. 2015;44(2):126-133.

32. Yi J, Pan BZ, Xiong L, Song HZ. Clinical significance of angiopoietin-like protein 4 expression in tissue and serum of esophageal squamous cell carcinoma patients. Med Oncol. 2013;30(3):680.

33. Shibata K, Nakayama T, Hirakawa H, Hidaka S, Nagayasu T. Clinicopathological significance of angiopoietin-like protein 4 expression in oesophageal squamous cell carcinoma. J Clin Pathol. 2010;63(12):1054-1058.

34. Wang Z, Han B, Zhang Z, Pan J, Xia H. Expression of angiopoietin-like 4 and tenascin $\mathrm{C}$ but not cathepsin $\mathrm{C}$ mRNA predicts prognosis of oral tongue squamous cell carcinoma. Biomarkers. 2010;15(1):39-46.

35. Hu J, et al. Angiopoietin-like 4: a novel molecular hallmark in oral Kaposi's sarcoma. Oral Oncol. 2011;47(5):371-375.

36. Dong D, Jia L, Zhou Y, Ren L, Li J, Zhang J. Serum level of ANGPTL4 as a potential biomarker in renal cell carcinoma. Urol Oncol. 2017;35(5):279-285.

37. Verine J, et al. Determination of angptl $4 \mathrm{mRNA}$ as a diagnostic marker of primary and metastatic clear cell renal-cell carcinoma. PLoS One. 2010;5(4):e10421.

38. Babapoor-Farrokhran S, et al. Angiopoietin-like 4 is a potent angiogenic factor and a novel therapeutic target for patients with proliferative diabetic retinopathy. Proc Natl Acad Sci U S A. 2015;112(23):E3030-E3039.

39. Jee $\mathrm{K}$, et al. Expression of the angiogenic mediator, angiopoietin-like 4 , in the eyes of patients with proliferative sickle retinopathy. PLoS One. 2017;12(8):e0183320.

40. Babapoor-Farrokhran S, Poon D, Solomon S, Montaner S, Sodhi A. Expression of angiogenic mediators in a patient with a retinal artery occlusion. Can J Ophthalmol. 2017;52(4):e122-e125.

41. Crawford TN, Alfaro DV, Kerrison JB, Jablon EP. Diabetic retinopathy and angiogenesis. Curr Diabetes Rev. 2009;5(1):8-13.

42. Miller JW, Le Couter J, Strauss EC, Ferrara
$\mathrm{N}$. Vascular endothelial growth factor a in intraocular vascular disease. Ophthalmology. 2013;120(1):106-114.

43. Ajlan RS, Silva PS, Sun JK. Vascular endothelial growth factor and diabetic retinal disease. Semin Ophthalmol. 2016;31(1-2):40-48.

44. Krispel C, Rodrigues M, Xin X, Sodhi A. Ranibizumab in diabetic macular edema. World J Diabetes. 2013;4(6):310-318.

45. Sodhi A, Montaner S. Angiopoietin-like 4 as an emerging therapeutic target for diabetic eye disease. JAMA Ophthalmol. 2015;133(12):1375-1376.

46. Ruia S, Saxena S, Gemmy Cheung CM, Gilhotra JS, Lai TY. Spectral domain optical coherence tomography features and classification systems for diabetic macular edema: a review. Asia Pac J Ophthalmol (Phila). 2016;5(5):360-367.

47. Robinson R, Barathi VA, Chaurasia SS, Wong TY, Kern TS. Update on animal models of diabetic retinopathy: from molecular approaches to mice and higher mammals. Dis Model Mech. 2012;5(4):444-456.

48. Hall A. Rho family GTPases. Biochem Soc Trans. 2012;40(6):1378-1382.

49. Gavard J, Gutkind JS. Protein kinase C-related kinase and ROCK are required for thrombininduced endothelial cell permeability downstream from Galpha12/13 and Galpha11/q. J Biol Chem. 2008;283(44):29888-29896.

50. Ferrara N. The role of VEGF in the regulation of physiological and pathological angiogenesis. EXS. 2005;(94):209-231.

51. Ferrara N. Vascular endothelial growth factor. Arterioscler Thromb Vasc Biol. 2009;29(6):789-791.

52. Simons M, Gordon E, Claesson-Welsh L. Mechanisms and regulation of endothelial VEGF receptor signalling. Nat Rev Mol Cell Biol. 2016;17(10):611-625.

53. Goh YY, et al. Angiopoietin-like 4 interacts with integrins beta1 and beta5 to modulate keratinocyte migration. Am J Pathol. 2010;177(6):2791-2803.

54. Gomez Perdiguero E, et al. ANGPTL4- $\alpha v \beta 3$ interaction counteracts hypoxia-induced vascular permeability by modulating Src signalling downstream of vascular endothelial growth factor receptor 2.J Pathol. 2016;240(4):461-471.

55. Parker MW, Guo HF, Li X, Linkugel AD, Vander Kooi CW. Function of members of the neuropilin family as essential pleiotropic cell surface receptors. Biochemistry. 2012;51(47):9437-9446.

56. Guo HF, Vander Kooi CW. Neuropilin functions as an essential cell surface receptor. J Biol Chem. 2015;290(49):29120-29126.

57. Soker S, Miao HQ, Nomi M, Takashima S, Klagsbrun M. VEGF165 mediates formation of complexes containing VEGFR-2 and neuropilin-1 that enhance VEGF165-receptor binding. J Cell Biochem. 2002;85(2):357-368.

58. Becker PM, et al. Neuropilin-1 regulates vascular endothelial growth factor-mediated endothelial permeability. Circ Res. 2005;96(12):1257-1265.

59. Lambert S, et al. HTLV-1 uses HSPG and neuropilin-1 for entry by molecular mimicry of VEGF165. Blood. 2009;113(21):5176-5185.

60. Guo HF, Li X, Parker MW, Waltenberger J, Becker $\mathrm{PM}$, Vander Kooi CW. Mechanistic basis for the potent anti-angiogenic activity of semaphorin $3 \mathrm{~F}$. Biochemistry. 2013;52(43):7551-7558.
61. Wang HB, et al. Neuropilin 1 is an entry factor that promotes EBV infection of nasopharyngeal epithelial cells. Nat Commun. 2015;6:6240.

62. Chomel C, et al. Interaction of the coiled-coil domain with glycosaminoglycans protects angiopoietin-like 4 from proteolysis and regulates its antiangiogenic activity. FASEB $J$. 2009;23(3):940-949.

63. Ip MS, Domalpally A, Sun JK, Ehrlich JS. Longterm effects of therapy with ranibizumab on diabetic retinopathy severity and baseline risk factors for worsening retinopathy. Ophthalmology. 2015;122(2):367-374.

64. Tjeerdema N, et al. Inflammation increases plasma angiopoietin-like protein 4 in patients with the metabolic syndrome and type 2 diabetes. BMJ Open Diabetes Res Care. 2014;2(1):e000034.

65. Lu Q, Lu P, Chen W, Lu L, Zheng Z. ANGPTL-4 induces diabetic retinal inflammation by activating Profilin-1. Exp Eye Res. 2018;166:140-150.

66. Yang YH, et al. Suppression of the Raf/MEK/ ERK signaling cascade and inhibition of angiogenesis by the carboxyl terminus of angiopoietin-like protein 4. Arterioscler Thromb Vasc Biol. 2008;28(5):835-840.

67. Ito $\mathrm{Y}$, et al. Inhibition of angiogenesis and vascular leakiness by angiopoietin-related protein 4 . Cancer Res. 2003;63(20):6651-6657.

68. Galaup A, et al. Angiopoietin-like 4 prevents metastasis through inhibition of vascular permeability and tumor cell motility and invasiveness. Proc Natl Acad Sci US A. 2006;103(49):18721-18726.

69. Bouleti C, et al. Protective effects of angiopoietin-like 4 on cerebrovascular and functional damages in ischaemic stroke. Eur Heart J. 2013;34(47):3657-3668.

70. Galaup A, et al. Protection against myocardial infarction and no-reflow through preservation of vascular integrity by angiopoietin-like 4 . Circulation. 2012;125(1):140-149.

71. Perdiguero EG, et al. Alteration of developmental and pathological retinal angiogenesis in angptl4-deficient mice. J Biol Chem. 2011;286(42):36841-36851.

72. Chung AS, Ferrara N. Developmental and pathological angiogenesis. Annu Rev Cell Dev Biol. 2011;27:563-584.

73. Crawford Y, Ferrara N. VEGF inhibition: insights from preclinical and clinical studies. Cell Tissue Res. 2009;335(1):261-269.

74. Akishima-Fukasawa Y, et al. Histopathological predictors of regional lymph node metastasis at the invasive front in early colorectal cancer. Histopathology. 2011;59(3):470-481.

75. Zhu P, et al. Angiopoietin-like 4 protein elevates the prosurvival intracellular $\mathrm{O} 2(-): \mathrm{H} 2 \mathrm{O} 2$ ratio and confers anoikis resistance to tumors. Cancer Cell. 2011;19(3):401-415.

76. Izraely S, et al. The metastatic microenvironment: brain-residing melanoma metastasis and dormant micrometastasis. Int J Cancer. 2012;131(5):1071-1082.

77. Yokouchi H, et al. Angiopoietin-like protein 4 (ANGPTL4) is induced by high glucose in retinal pigment epithelial cells and exhibits potent angiogenic activity on retinal endothelial cells. Acta Ophthalmol. 2013;91(4):e289-e297. 
78. Capozzi ME, McCollum GW, Savage SR, Penn JS. Peroxisome proliferator-activated receptor- $\beta / \delta$ regulates angiogenic cell behaviors and oxygen-induced retinopathy. Invest Ophthalmol Vis Sci. 2013;54(6):4197-4207.

79. Lu Q, Zou W, Chen B, Zou C, Zhao M, Zheng Z. ANGPTL-4 correlates with vascular endothelial growth factor in patients with proliferative diabetic retinopathy. Graefes Arch Clin Exp Ophthalmol. 2016;254(7):1281-1288.

80. Kwon SH, Shin JP, Kim IT, Park DH. Aqueous Levels of Angiopoietin-like 4 and semaphorin 3e correlate with nonperfusion area and macular volume in diabetic retinopathy. Ophthalmology. 2015;122(5):968-975.

81. Kim JH, Shin JP, Kim IT, Park DH. Aqueous angiopoietin-like 4 levels correlate with nonperfusion area and macular edema in branch retinal vein occlusion. Invest Ophthalmol Vis Sci. 2016;57(1):6-11.

82. Kim JH, Shin JP, Kim IT, Park DH. Angiopoietinlike 4 correlates with response to intravitreal ranibizumab injections in neovascular age-related macular degeneration. Retina (Philadelphia, Pa). 2018;38(3):523-530.

83. Meng Q, et al. Hypoxia-inducible factor-dependent expression of angiopoietin-like 4 by conjunctival epithelial cells promotes the angiogenic phenotype of pterygia. Invest Ophthalmol Vis Sci. 2017;58(11):4514-4523.

84. Raja SG. Evaluation of clinical efficacy of fasudil for the treatment of pulmonary arterial hypertension. Recent Pat Cardiovasc Drug Discov. 2012;7(2):100-104.

85. Wu FQ, Zhu SY, He CX, Gu MX. [Effects of fasudil on the expression of Rho kinase-1 and airway inflammation in a mouse model of asthma]. Zhonghua Jie He He Hu Xi Za Zhi. 2009;32(11):847-849.

86. Babelova A, et al. Activation of Rac-1 and RhoA contributes to podocyte injury in chronic kidney disease. PLoS One. 2013;8(11):e80328.

87. Fujimura M, Usuki F, Kawamura M, Izumo S. Inhibition of the Rho/ROCK pathway prevents neuronal degeneration in vitro and in vivo following methylmercury exposure. Toxicol Appl Pharmacol. 2011;250(1):1-9.

88. Sanjari N, et al. Intravitreal injection of a rhokinase inhibitor (fasudil) for recent-onset nonarteritic anterior ischemic optic neuropathy. JClin Pharmacol. 2016;56(6):749-753.

89. Defert O, Boland S. Rho kinase inhibitors: a patent review (2014 - 2016). Expert Opin Ther Pat. 2017;27(4):507-515.

90. Arita $\mathrm{R}$, et al. Rho kinase inhibition by fasudil ameliorates diabetes-induced microvascular damage. Diabetes. 2009;58(1):215-226.

91. Song H, Gao D. Fasudil, a Rho-associated protein kinase inhibitor, attenuates retinal ischemia and reperfusion injury in rats. Int J Mol Med. 2011;28(2):193-198.

92. Rothschild PR, et al. ROCK-1 mediates diabetes- induced retinal pigment epithelial and endothelial cell blebbing: Contribution to diabetic retinopathy. Sci Rep. 2017;7(1):8834.

93. Kawasaki T, et al. A requirement for neuropilin-1 in embryonic vessel formation. Development. 1999;126(21):4895-4902.

94. Yuan L, et al. Abnormal lymphatic vessel development in neuropilin 2 mutant mice. Development. 2002;129(20):4797-4806.

95. Kärpänen T, et al. Functional interaction of VEGF-C and VEGF-D with neuropilin receptors. FASEB J. 2006;20(9):1462-1472.

96. Takashima $S$, et al. Targeting of both mouse neuropilin-1 and neuropilin-2 genes severely impairs developmental yolk sac and embryonic angiogenesis. Proc Natl Acad Sci U S A. 2002;99(6):3657-3662.

97. Cerani A, et al. Neuron-derived semaphorin 3A is an early inducer of vascular permeability in diabetic retinopathy via neuropilin-1. Cell Metab. 2013;18(4):505-518.

98. Shen J, et al. Deficiency of neuropilin 2 suppresses VEGF-induced retinal neovascularization. $\mathrm{Mol}$ Med. 2004;10(1-6):12-18.

99. Gavard J, Gutkind JS. VEGF controls endothelialcell permeability by promoting the beta-arrestindependent endocytosis of VE-cadherin. Nat Cell Biol. 2006;8(11):1223-1234.

100.Faye C, et al. Molecular interplay between endostatin, integrins, and heparan sulfate. J Biol Chem. 2009;284(33):22029-22040. 